\title{
Biomarkers of meat and seafood intake: an extensive literature review
}

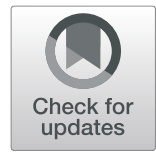

\author{
Cătălina Cuparencu ${ }^{1 * \dagger} \mathbb{B}$, Giulia Praticó ${ }^{1 \dagger}$, Lieselot Y. Hemeryck ${ }^{2 \dagger}$, Pedapati S. C. Sri Harsha ${ }^{3 \dagger}$, Stefania Noerman ${ }^{4}$, \\ Caroline Rombouts ${ }^{2}$, Muyao Xi ${ }^{1}$, Lynn Vanhaecke ${ }^{2}$, Kati Hanhineva ${ }^{4}$, Lorraine Brennan ${ }^{3}$ and Lars O. Dragsted ${ }^{1}$
}

\begin{abstract}
Meat, including fish and shellfish, represents a valuable constituent of most balanced diets. Consumption of different types of meat and fish has been associated with both beneficial and adverse health effects. While white meats and fish are generally associated with positive health outcomes, red and especially processed meats have been associated with colorectal cancer and other diseases. The contribution of these foods to the development or prevention of chronic diseases is still not fully elucidated. One of the main problems is the difficulty in properly evaluating meat intake, as the existing self-reporting tools for dietary assessment may be imprecise and therefore affected by systematic and random errors. Dietary biomarkers measured in biological fluids have been proposed as possible objective measurements of the actual intake of specific foods and as a support for classical assessment methods. Good biomarkers for meat intake should reflect total dietary intake of meat, independent of source or processing and should be able to differentiate meat consumption from that of other protein-rich foods; alternatively, meat intake biomarkers should be specific to each of the different meat sources (e.g., red vs. white; fish, bird, or mammal) and/or cooking methods. In this paper, we present a systematic investigation of the scientific literature while providing a comprehensive overview of the possible biomarker(s) for the intake of different types of meat, including fish and shellfish, and processed and heated meats according to published guidelines for biomarker reviews (BFIrev). The most promising biomarkers are further validated for their usefulness for dietary assessment by published validation criteria.
\end{abstract}

Keywords: Biomarkers of food intake, Protein sources, Red meat, Processed meat, Poultry, Fish, Seafood

\section{Background}

Meat, including fish and shellfish, represents a valuable constituent of a balanced omnivorous diet. The importance of meat from a nutritional point of view is related to its high-quality protein content, as it comprises a balanced source of all essential amino acids for muscle maintenance [1]. Minerals and vitamins, such as iron and $\mathrm{B}_{12}$ vitamin, and other micronutrients that are essential for growth and development, are additionally highly bioavailable from meat compared to other sources [2]. Meat processing, such as curing, smoking, or heating, further improves its organoleptic properties, as well as its microbiological safety and shelf life [3]. On the

\footnotetext{
* Correspondence: cup@nexs.ku.dk

${ }^{\dagger}$ Cătălina Cuparencu, Giulia Praticó, Lieselot Y. Hemeryck, and Pedapati S.C.

Sri Harsha shared first co-authorship.

'Department of Nutrition, Exercise and Sports, University of Copenhagen,

Rolighedsvej 30, 1958 Frederiksberg C, Denmark

Full list of author information is available at the end of the article
}

other hand, high consumption of processed meat and possibly red meat has been associated with a series of adverse health outcomes, including cardiovascular disease (CVD) [4], overall mortality [5], and certain types of cancer, especially colorectal cancer [6-8]. However, recent large, prospective investigation including almost half a million subjects from ten European countries [9], only found significant associations with processed meat intake. Poultry was not related to all-cause mortality, whereas red meat was related to higher all-cause mortality only before correcting for potential confounding [9]. Additionally, high intake of red and processed meat has also been associated with an increased risk of developing type 2 diabetes, although major inconsistencies regarding consumption levels exist between studies [10-12]. Fish and shellfish intake has been associated with positive health outcomes, such as decreasing CVD risk [13] and possibly colorectal cancer $[14,15]$, while its role in 
preventing type 2 diabetes development is still unclear $[16,17]$. Different outcomes in the studies may also associate with the type of fish consumed and the cooking methods [17]. Moreover, aquatic meat has proven to be one of the major dietary sources of contaminants that are potentially harmful to human health, such as methylmercury and arsenic $[18,19]$.

Since associations are always debatable in terms of cause and effect and weak associations like those between meat and chronic disease are always at a high risk of being affected by bias or confounding, it is important to find biomarkers able to objectively discriminate between different classes of meat, particularly red meat, white meat from poultry, white meat from fish or shellfish, and processed meats [20]. The existing selfreporting tools for dietary assessment, such as food frequency questionnaires (FFQ) and dietary records, are imprecise and can be affected by systematic and random errors, especially when a certain food is perceived as healthy or unhealthy [21]. Dietary biomarkers have been proposed as possible objective measurements of the actual specific food intakes or at least as a support for the classical assessment methods [22]. A good marker of overall meat intake should reflect total dietary meat intake differentiating any meat consumption from that of other protein-rich foods; additional markers should discriminate between different meat sources (e.g., red vs. white or between different species, e.g., pork vs. beef) and identify cooking methods. In this paper, we present the results of a systematic search of the scientific literature according to the BFIrev methodology [23] providing a comprehensive overview of the possible marker(s) for the intake of different meat types, including fish and shellfish. The most promising biomarkers are further validated according to a validation scheme previously proposed for biomarkers of food intake [24]. This review represents the continuation of the reviewing process for candidate intake biomarkers for various foods of animal origin initiated in a previous paper on dairy and egg biomarkers, as part of the FoodBAll project [25].

\section{Methods}

\section{Selection of food groups}

In order to obtain a good coverage of the different meat sources, meat was subdivided into fresh meat (e.g., overall meat intake, red meat, white meat), fish and fish oil, and other aquatic meat, e.g., various shellfish, processed meat products (cured and smoked), and offal or organ meats. Two particular groups, strongly heated (e.g., grilled) meat products and biomarkers for fish contaminants, were also investigated. A total of nine food groups were thus selected for reviewing their respective markers of intake. A systematic literature search was carried out separately for each food group as detailed below.

\section{Primary literature search}

The reviewing process, including article search and selection, reviewing and reporting of the results, follows the guidelines previously proposed by the FoodBAll consortium to carry out an extensive literature search and evaluation of biomarkers for food intake (BFIs) [23].

In brief, original research papers and reviews were searched in at least two databases, among which PubMed, Scopus, and ISI Web of Knowledge, using combinations of the grouped search terms (biomarker* OR marker* OR metabolite* OR biokinetics OR biotransformation) AND (trial OR experiment OR study OR intervention OR cohort) AND (human* OR men OR women OR patient* OR volunteer* OR participant*) AND (urine OR plasma OR serum OR blood OR excretion) AND (intake OR meal OR diet OR ingestion OR consumption OR eating OR drink* OR administration), as reported in Additional file 1: Table S1, together with specific keywords related to each animal-derived food group (Additional file 1: Table S2). The fields used as default for each of the databases were [All Fields] for PubMed, [Article Title/ Abstract/ Keywords] for Scopus, and [Topic] for ISI Web of Science, respectively. For almost all food groups, the three databases were consulted. For "processed meat" and "offal meat," the search was carried out only in PubMed and ISI Web of Knowledge. The literature search process was carried out between November and December 2015 and updated at the end of December 2018 for all food groups.

The search was limited to papers written in English, while no restriction was applied regarding publication date. The research papers identifying or using potential biomarkers of intake for the different kinds of meat were selected from the list of retrieved references by one or more skilled researchers in a process outlined in Additional file 1: Figure S1. The papers obtained from the search in different databases were merged and filtered for duplicates. Subsequently, papers were screened based on title and abstract. The selected papers were then retrieved and assessed for eligibility based on the contents of the whole manuscript. Additional papers were identified from reference lists in these papers and from reviews or book chapters identified through the search. The result was a list of compounds potentially relevant as biomarkers for the food group and corresponding references.

\section{Secondary search, marker identification, and classification} A second search step was used to evaluate the apparent specificity of the markers in the list. The compound databases HMDB [26] and FooDB (http://foodb.ca/)) were used for a first evaluation of marker specificity. If the marker was not specific for a single food group, it was noted whether it was related to any food outside the meat food groups. In the latter case, unless levels were 
reported to be very low elsewhere, the compound was omitted from the list for the next search. The remaining list of putative biomarkers was used for a second literature search in the three bibliographic databases used also for the primary search. This was done to potentially identify other foods containing the putative biomarkers or their precursors as well as foods otherwise associated with these compounds. For the second web-search, we used the chemical name(s) of the marker as keyword, together with AND (biomarker* OR marker* OR metabolite* $^{*}$ OR biokinetics OR biotransformation). Further filters, such as (urine OR plasma OR serum OR blood OR excretion) AND (intake OR meal OR diet OR ingestion OR consumption OR eating OR drink* OR administration) AND (human* OR men OR women OR patient* OR volunteer* OR participant* OR subject*), were added based on the results obtained. Again, markers not related to meat intake were deleted from the list. The remaining putative markers with potential specificity for each food group or for several combined animal food groups were finally listed in Additional file 2: Table S3, together with information about the study designs of the papers reporting their use. Due to the high number of results on marine fatty acids from the search on "fish and fish oil," only the most representative among the observational studies having the highest number of participants were kept in Additional file 2: Table S3. A summary of the markers considered for further validation according to the validation guidelines [24] is reported in Additional file 3: Table S4.

\section{Marker validation}

In order to further assess the validity of the candidate biomarker, the scoring system for biomarkers of food intake was used as described previously [24]. Briefly, the usefulness of each selected marker in Additional file 3: Table S4 has been established by answering a set of simple questions reflecting the biological and analytical criteria that a biomarker should fulfill in order to be considered valid. The questions have been answered for the most promising biomarkers. Possible answers were $\mathrm{Y}$ (yes), N (no), or U (unknown or uncertain). Each candidate marker was evaluated for plausibility (question 1), meaning that it is considered a plausible BFI for the food or food group based on food chemistry; dose-response relationship between quantity of food ingested and biomarker response (question 2); kinetics of immediate postprandial response (question 3a) and/or of repeated intakes (question 3b); robustness in complex diets or real-life exposure situations (question 4); reliability, i.e., concordance with other established measures of intake for the food or food group in question (question 5); analytical aspects, including the chemical stability of the marker (question 6), its analytical performance (question 7); and reproducibility in different labs (question 8) [24].

\section{Results \\ General biomarkers of meat intake}

General biomarkers of meat intake are common to all or a large number of foods investigated in this review. The search for biomarkers of meat intake provided 953 hits after removal of duplicates, resulting in the final selection of 20 papers from the web-search (Additional file 1: Figure S1). From the analysis of the reference lists and from the secondary search, another six papers were included in the review, resulting in a total of 26 papers; several relevant papers were dealing with specific meat subgroups and were therefore moved to this heading after reading the full text papers (Additional file 2: Table S3). The main markers associated with the intake of animal protein from meats were various isotope ratios, anserine, carnosine, 1- and 3-methylhistidine (MH), creatine, creatinine, carnitine and acylcarnitines, taurine, trimethylamine oxide (TMAO), and several unidentified features specified by their $\mathrm{m} / \mathrm{z}$ ratio. In addition, urinary nitrogen was used for total protein intake assessment in many studies but the marker is not a specific meat intake biomarker and therefore not included. The main human sample investigated in the studies was urine, followed by plasma, although feces and hair were also used occasionally. Both interventional and observational studies were considered for the evaluation of the putative BFIs for meat intake.

\section{Histidine-related compounds}

Vertebrate muscles contain a large amount of dipeptides containing histidine, such as carnosine ( $\beta$-Alanyl-L-histidine), anserine ( $\beta$-Alanyl-3-methyl-L-histidine), and balenine ( $\beta$-Alanyl-1-methyl-histidine); therefore, the quantification in human bio-specimens of such compounds or products of their catabolism, such as $\beta$-alanine, 1-MH $\left(\mathrm{N}^{\mathrm{T}}-\mathrm{MH}\right)$ and $3-\mathrm{MH}$ $\left(\mathrm{N}^{\mathrm{T}}-\mathrm{MH}\right)$, may support the assessment of meat intake [20]. Some degree of confusion exists regarding the nomenclature of 1-MH and 3-MH in regards to the numbering of the nitrogen in the imidazole ring of the histidine moiety because chemists and biochemists have used opposite designations for the two molecules; for instance, PubChem lists both compounds with both names and with both synonyms. We use here the chemical nomenclature, where $\mathrm{N}^{\mathrm{\pi}}-\mathrm{MH}$ ( $\pi$ for nearest to the side chain) is termed 3-methylhistidine, while the common form in human muscle, $\mathrm{N}^{\mathrm{T}}-\mathrm{MH}$ ( $\mathrm{t}$ for far from the side chain) is termed, 1-methylhistidine, as recommended in the IUPAC definition and in the major compound databases $[26,27]$. The synonym of the dipeptide anserine is thus $\beta$-Alanyl-3-methyl-L-histidine (Mora et al. 2007; HMDB metabocard for anserine (HMDB00194)); however, while some older papers have termed it $\beta$-Alanyl-1- 
methyl-L-histidine, in this review we have reported the results from these papers with the IUPAC name [28, 29]. The dipeptide anserine, common in poultry, undergoes cleavage to give rise to $3-\mathrm{MH}$. The dipeptide balenine, common in some whales, cleaves to form 1-MH [30].

The content of histidine-containing dipeptides in food is highly variable [31-33] and their use as markers for meat intake may therefore be better at the group level than at the individual level. Carnosine contents are higher in beef and pork, while they are slightly lower in poultry $[31,33]$ and almost absent in fish, with the exception of some species of Anguilloidei (eel) [32]. Moreover, this compound was not detected in other foods of animal origin, such as milk [34] and may only be found in some sources of liver $[35,36]$, suggesting that the compound could represent a putative marker to assess the intake of terrestrial meat. Carnosine levels were found to be markedly increased in urine after the ingestion of beef, pork, chicken, and eel [31, 34, 37, 38], showing also doseresponse [34]. Cheung et al. [38] further compared several meat sources and reported that urinary carnosine associates with meat intake in a selected group of subjects from the European Prospective Investigation into Cancer and Nutrition (EPIC). Carnosine levels were significantly higher in the urine samples from subjects who consumed chicken, red meat, and processed meat compared to those consuming fish, with no significant differences observed between subjects consuming similar amounts of the three types of meat. Carnosine is completely excreted in urine within $20-25 \mathrm{~h}$ following the meal, reaching a peak after 5 $\mathrm{h}$ [31]. This kinetic behavior makes the compound suitable for quantification in $24 \mathrm{~h}$ urines to estimate total intake of terrestrial muscle meat.

The results regarding the detection of carnosine in plasma are contradictory. Parker et al. [39] observed a peak in plasma at $2.5 \mathrm{~h}$ after beef consumption returning to background levels after $5.5 \mathrm{~h}$, while other studies failed to confirm this $[37,38]$. In a cross-sectional study of 294 Bavarian men and women completing 24-h recalls and providing non-fasting samples, plasma carnosine was associated with intake levels for all meat, red meat, and beef + pork but not for poultry, fish, or dairy [40] with approximately similar intake levels. These discrepancies could therefore be ascribed to a possibly weak performance of this marker, even at the group level. Plasma carnosine needs to be further investigated in carefully controlled trials to evaluate its usefulness as a BFI. With the current evidence, plasma carnosine is not a likely candidate marker for meat intake.

Anserine is present in the muscles of different nonhuman vertebrates, with poultry, rabbit, tuna, plaice, and salmon having generally higher contents than other marine foods, beef, or pork [31-33, 41]. An increase of urinary anserine excretion was found in humans after the consumption of chicken [31,37,38], rabbit [41], and tuna [31] and has been associated with intake of chicken [38], salmon [42], and, to a lesser extent, beef [34], while other observational evidence indicates no increase with fish intake [38]. In particular, anserine in urine was a good marker for chicken intake based on FFQ data in a selected sample of high vs. non-consumers from the EPIC study [38].

In a cross-sectional study from Bavaria, plasma anserine was associated in a dose-response manner with 24-h recall information for total meat, beef + pork, turkey, processed meat, and total dairy but not with chicken, total poultry, "seafood," or beef or pork, individually [40].

In the human body, anserine is a substrate for carnosinase and is mainly degraded and excreted as 3-MH [31]. Endogenous formation of $3-\mathrm{MH}$ is minimal in humans; therefore, plasma and urinary 3-MH are primarily associated with food intake. A significant increase in the level of urinary 3-MH was observed after consumption of red meat $[34,43,44]$, chicken or poultry [31, 34, 38, 44-46], and fish $[31,38,42,44,47]$, with a clear dose- or time-response relationship between urinary 3-MH excretion and the intake of the different animal proteins [38, 43, 44, 46]. Urinary 3-MH was able to discriminate subjects with vegetarian or omnivorous diets in controlled settings [43] as well as in free living subjects [48], suggesting that this marker could provide evidence of overall habitual meat intake. Importantly, the excretion of 3-MH depends on meat source. In studies reflecting recent intake, poultry intake gave higher levels of 3-MH when compared to those following consumption of pork and/or beef, most likely due to anserine breakdown $[34,38,44]$. The discrimination between poultry and fish intake is more uncertain, as fish species vary significantly in anserine and 3-MH content [32, 44], but chicken meat seems to have generally higher levels than fish. An increased level of 3-MH was reported after consumption of chicken compared to that of cod [38]. In one observational study from the Adventist Health Study-2 cohort $(n=1011$ subjects), urinary $3-\mathrm{MH}$ was positively associated with the intake of animal protein, red meat, and poultry, assessed both by FFQ and 24-h recall, while the correlation tended to be lower with fish, probably due to the preference for meat consumption in the population investigated [49]. On the other hand, Cheung et al. [38] observed higher concentration of 3-MH in the urine of a subset of EPIC subjects who consumed fish (mainly cod and haddock) compared to those consuming red and processed meat. Also in this case, poultry intake gave the highest amount of 3-MH excretion. 3-MH peaks in urine around $5 \mathrm{~h}$ after intake and decreases to baseline levels after $\sim 40 \mathrm{~h}$ [31] or $48 \mathrm{~h}$ [50]. Sjolin et al. [44] reported an excretion half-life of $11.7 \mathrm{~h}$ for $3-\mathrm{MH}$, indicating that excretion would not be complete in a $24-\mathrm{h}$ urine collection. In a recent study, ten healthy subjects ingested increasing amounts of chicken daily for 3 days in 
three consecutive weeks. Peak postprandial plasma levels on day 3 and fasting levels in urine and plasma on day 4 in each week increased with dose [46] corroborating accumulation of 3-MH with daily intakes. 3-MH was also detected in plasma samples after the intake of chicken and to a lesser extent after the consumption of fish in another controlled intervention study [38]. A much smaller increase was also observed after intake of beef and processed meat. Urinary and plasma 3-MH therefore represent possible markers to assess meat intake, but due to the differences between meat sources with higher levels observed after intake of chicken and some species of fish, the marker should be carefully evaluated for different study populations, based on their food preferences.

1-MH is a constituent of actin and myosin, the contractile proteins of skeletal muscles, and its urinary excretion is rapid following muscle protein breakdown [51]. This compound has been observed to significantly increase in urine after meat consumption [43, 52-54]. A single meal with ${ }^{15} \mathrm{~N}$-labeled beef increased ${ }^{15} \mathrm{~N}$-labeled $1-\mathrm{MH}$ in urine at $0-8 \mathrm{~h}$, confirming that food-derived $1-\mathrm{MH}$ is excreted in urine [55]. Unlike 3-MH, the excretion of 1-MH increased during $72 \mathrm{~h}$ of fasting indicating catabolism of muscle tissue [56]. Daily meat intake in omnivores associates with 1-MH excretion, however with much more variation compared to 3-MH [34, 44], although with a similar postprandial kinetics and a half-life of $12.6 \mathrm{~h}$ [44]. Endogenous production causes a high inter-individual variability making this marker less suitable to asses meat intake by itself [43]. In one observational study, urinary 1-MH after meat and fish intake was not different between high and nonconsumers [38]. Plasma 1-MH was not associated with any group of meat, poultry, or fish intake in a study based on 24-h recalls [40] and did not increase after a meal intervention with chicken breast [50].

As a consequence of the breakdown of histidine-containing dipeptides, $\beta$-alanine can also be observed in body fluids [41]. So far, only one metabolomics study reported an increase in plasma levels of this compound after beef consumption [57]. In an early study, $\beta$-alanine appeared to increase in human urine only after an anserine containing meal [41], but since $\beta$ alanine can also be formed endogenously by catabolism of pyrimidines [26], it may not be a robust marker to assess meat intake; further investigations are necessary to evaluate its specificity and sensitivity in a cross-sectional study.

\section{Carnitine and acylcarnitines}

Carnitine represents an essential cofactor in fatty acid metabolism in mammals, as it transports fatty acids under the form of acylcarnitines into the mitochondria of muscle cells for cellular energy production by lipid $\beta$ oxidation [58]. Carnitine may be synthetized in the body from the essential amino acids lysine and methionine. Most of the carnitine in omnivores comes from dietary sources, particularly beef and lamb [20]. Carnitine and acetylcarnitine were higher in the urine [59] and serum [60] of subjects consuming high-meat diets compared to vegetarians, and their urinary values were representative of habitual intake of red and processed meat in freeliving subjects [61, 62]. Three acylcarnitines (acetylcarnitine, propionylcarnitine and 2-methylbutylcarnitine) were significantly higher in the urine of subjects from the EPIC cohort after the intake of meat and fish compared to control subjects with no meat intake, with no distinction among the different animal protein sources [38]. Propionylcarnitine and acetylcarnitine were also observed in plasma after a dietary intervention with meat and fish from the same research group [38]. Acetylcarnitine had slower kinetics than propionylcarnitine, reaching the highest value after $24 \mathrm{~h}$ when propionylcarnitine had already returned to the baseline level. In the EPIC-Oxford cohort, a series of acylcarnitines were also characteristic of the dietary pattern associated with high meat intake [63]. In particular, the concentrations of carnitine and acylcarnitines C-4 and C-5 were highest in meat eaters, followed by fish eaters, vegetarians, and vegans. Furthermore, $\mathrm{C}-3$ and $\mathrm{C}-16$ were higher in meat eaters and lower in vegans. Carnitine and acylcarnitines may reflect the intake of highly accessible amino acids and fatty acids contained in meat and fish and may be considered generic markers of intake of foods of animal origin. However, given that physiological conditions such as age, gender, and health status, as well as the intake of other foods with highly accessible amino acids or fatty acids may affect plasma acylcarnitine levels and their excretion into urine $[64,65]$, these metabolites may not be suitable markers per se for specific and quantitative assessment of meat intake and are therefore not considered for further validation as single markers.

\section{Creatine and creatinine}

Creatine is present in animal muscles, mainly in the form of phosphocreatine, and can be synthesized endogenously from arginine, glycine, and methionine. The main dietary source is meat, including red meat, fish, and poultry [66]. Several studies reported an increase in creatine levels after meat intake in urine [59, 62, 67, 68], erythrocytes [60], plasma, and serum [60, 69, 70]. Even a single meal containing meat has been shown to increase creatine in urine [71]. Associations between urinary creatine and habitual intake of shellfish [62] and oily fish such as salmon [42] have also been reported. Pallister et al. [69] proposed circulating levels of creatine as a possible marker of habitual intake of red meat and poultry, as observed in the UK Twin cohort study ( $n=$ 3559 subjects). However, experimental studies supporting this observation are lacking. Creatine in urine and 
plasma/serum are therefore candidate markers of total meat intake.

Creatinine can originate both from spontaneous decomposition of creatine and creatine phosphate in the human body and to some extent also during cooking of meat [72]. An effect of diet on urinary creatinine was observed in a study comparing omnivores and vegetarians, being higher in the former group [60]. Cross et al. [43] showed that urinary creatinine performed as a food intake biomarker only for extreme levels of meat intake. Creatinine excretion mainly reflects endogenous production. The excretion into urine is highly regulated in the body and is proportional to the total content of creatine and creatine phosphate, i.e., the total muscle mass, in individuals with normal kidney function [20]. Therefore, creatinine cannot be used to estimate intake of meat without correcting for muscle mass and the compound is consequently not generally useful as a meat intake biomarker.

\section{Taurine}

Taurine is the most abundant free amino acid in animal tissues and mainly derives from the ingestion of many different foods of animal origin including eggs and dairy, while it can also be synthetized endogenously [73]. Metabolomics studies reported higher urinary taurine in omnivorous subjects compared to vegetarians [59, 74]. In two intervention studies, taurine excretion increased with animal protein intake but the change was not significant for low levels of meat intake [43, 75]. Taurine also associated with shellfish intake in an observational study ( $n=253$ subjects), but not with general meat intake [62]. The marker does not appear robust enough to assess total meat intake.

\section{Trans-4-hydroxyproline}

Hydroxyproline (Hyp) is a post-translationally modified amino acid that represents a major component in protein collagen and elastin, and its main dietary source comes from animal foods rich in connective tissue [76]. This compound was found in plasma after the intake of beef [57], while Pallister et al. [69, 70] observed a significant correlation between the level of this compound in fasting blood and the frequency of meat and processed meat consumption in the UK Twin cohort. High levels of collagen have also been reported in fish [77], therefore it can be speculated that this marker may better reflect the overall intake of meat rich in collagen, rather than that of red or processed meat. The dipeptide prolylhydroxyproline (Pro-Hyp) has been reported as a marker of a healthy diet rich in fish [78]. Since there is also collagen in offal meat, HyP in plasma is a potential intake marker of total meat intake. Further studies of the contribution of endogenously formed HyP to its urinary excretion is needed as well as studies on HyP containing dipeptides in plasma and urine to potentially discriminate between endogenous and dietary sources.

\section{$\delta 15 N$ and $\delta 13 C$}

Recently, the ratios of naturally occurring stable isotopes of carbon $\left({ }^{13} \mathrm{C} /{ }^{12} \mathrm{C}\right.$ ratio, expressed as $\left.\delta 13 \mathrm{C}\right)$ and of nitrogen $\left({ }^{15} \mathrm{~N} /{ }^{14} \mathrm{~N}\right.$ ratio, expressed as $\left.\delta 15 \mathrm{~N}\right)$ in biological fluids and tissues have been proposed as novel nutritional biomarkers of meat and fish intake. Such an approach was originally used in archeology and recently introduced also in nutritional science to support dietary assessment of animal proteins [79]. When animals consume plants, they incorporate carbon and nitrogen from plants into their own tissues. The abundance of $\delta 13 \mathrm{C}$ depends on the photosynthetic process utilized by the plants consumed by the animals; hence, animal feeding represents the primary determinant of variation in animal $\delta 13 C$ values [79]. In particular, C4 plants (crabgrass, sugarcane, and corn) have $\delta 13 \mathrm{C}$ values $12-13 \%$ higher than C3 plants (wheat, rice, and the majority of fruit and vegetables). Incorporation of ${ }^{15} \mathrm{~N}$ depends also on dietary sources. In particular, animals preferentially excrete ${ }^{14} \mathrm{~N}$ as waste nitrogen, leading to $\delta 15 \mathrm{~N}$ values that are $3 \%$ to $4 \%$ higher than in their diet [79]. Moreover, ${ }^{15} \mathrm{~N}$ abundance of tissue proteins increases in the food chain. As a consequence, animal-derived food proteins are expected to have a higher amount of ${ }^{15} \mathrm{~N}$ compared to plantderived protein food. In particular, the $\delta 15 \mathrm{~N}$ value has been suggested as a particularly good marker for aquatic meat consumption, as commonly consumed fish, such as tuna, salmon, and cod, are predators and reside at the higher levels of the trophic chain [80].

In a randomized $4 \times 8$ days cross-over study, Kuhnle and coworkers [81] observed significantly different isotope ratios among different diets (meat, fish, half-meathalf-fish, and vegetarian) in feces and urine samples, but not in blood samples, possibly due to the slower protein turnover. Higher $\delta 13 \mathrm{C}$ and $\delta 15 \mathrm{~N}$ were observed in urine and fecal samples following a fish diet, and lower following a vegetarian diet, where protein had been substituted by carbohydrates. It was not possible to distinguish between meat and half-meat-half-fish diets in any of the biofluids. The higher level of $\delta 13 \mathrm{C}$ and $\delta 15 \mathrm{~N}$ after the fish diet may be ascribed to the high level of fish that the subjects consumed during the intervention period. These results suggest that urinary and fecal $\delta 13 \mathrm{C}$ and $\delta 15 \mathrm{~N}$ may be suitable biomarkers to assess short-term meat and fish intake, while blood levels may reflect longer-term intake. This interpretation is supported by data from Patel et al. [82], who were able to distinguish vegetarian from non-vegetarian subjects by serum $\delta 13 \mathrm{C}$ and $\delta 15 \mathrm{~N}$ abundance in a sub-cohort within the EPICNorfolk study $(n=1254)$. Serum $\delta 13 \mathrm{C}$ and $\delta 15 \mathrm{~N}$ were positively associated $(P<0.001)$ with fish protein intake 
(assessed by FFQ) and increased with increased fish consumption, while animal proteins, including dairy and meat proteins, were significantly associated with $\delta 15 \mathrm{~N}$. Serum $\delta 15 \mathrm{~N}$ increased also with total animal protein consumption. Overall fish intake is more clearly associated with the isotope ratios in populations with high fish intakes while the ratios cannot distinguish different sources of intake in populations with mixed diets of aquatic and terrestrial meats [80, 83-85].

To monitor the habitual intake of animal proteins, $\delta 15 \mathrm{~N}$ and $\delta 13 \mathrm{C}$ were measured in hair and compared with the data collected from FFQ and dietary records in observational studies [86-89]. This is possible as hair keratin is not recycled in the body, and allows a reliable recording of dietary habits during the past months [86]. Overall $\delta 15 \mathrm{~N}$ and $\delta 13 \mathrm{C}$ in hair are positively associated with total meat intake and no specific meat group seems to be dominant, except when either fish or terrestrial meats dominate the diet $[86,90]$.

In conclusion, $\delta 15 \mathrm{~N}$ alone or in combination with $\delta 13 \mathrm{C}$ represent promising markers to assess animal protein as well as meat intake, both for short-term based on urine or feces and habitual intake based on blood and hair. As these ratios are affected by local diets and agriculture, they should be validated for each population before being applied.

\section{TMAO}

The intake of red meat has been mentioned as a cause of increased plasma or urine levels of TMAO in some observational studies [91, 92], while increased levels are characteristic of fish or shellfish in intervention studies. In cross-over randomized controlled trials (RCTs) comparing meals with beef, egg, fruit, and cod, urine or plasma levels increased only with cod [38, 93-95]. Plasma TMAO, trimethylamine (TMA), and dimethylamine increased quickly after cod with a peak at $2 \mathrm{~h}$. Beef and egg slightly increased postprandial plasma TMAO and the volunteers segregated into TMAO producers and non-producers, based on their microbiota [94]. In an intervention study comparing 4-week intakes of red meat, poultry, or non-meat proteins with levels of TMAO in plasma and urine, TMAO excretion as well as plasma levels increased only with red meat. A subset of 13 volunteers received isotope-labeled carnitine and betaine but only carnitine increased circulating isotopelabeled TMAO [96]. In conclusion, TMAO in urine does not seem to be a marker of recent red meat intake but is increased postprandially with certain types of fish (see section below). The microbial formation of TMA and its hepatic oxidation to TMAO increase plasma levels by 2-4-fold in subjects with a pre-disposing microbiota after diets rich in TMA precursors, including carnitine. However, plasma TMAO cannot serve as a marker of red meat intake because the response varies between individuals and may also depend on the efficiency of the kidney in removing it from the circulation.

\section{Mammalian (red) meat}

Red meat includes muscle meat from mammals, although the amount of color (heme) varies considerably between muscle tissues from different species. Sometimes, the term pink meat is used to include pork, which has lower contents of heme iron compared to beef, veal, and mutton. However, we have included pork with the mammalian red meats here. Although some non-muscle mammalian meats (e.g., liver) and some fish (e.g., tuna) and poultry (e.g., duck) have red meat, they are usually not included with the term and also not included in this category here. The primary literature search for mammalian red meat in the online databases (Web Of Science, Pubmed and Scopus) resulted in a total of 1516 articles after the removal of duplicates. The exclusion of animal studies, studies on nutrition, physiology, and food composition based on title, abstract, or full text reduced the number of articles to 49. After the evaluation of marker specificity in online databases (HMDB and FooDB) and from additional literature, 26 putative biomarkers (lead, indole propionate, xylitol, ethyl glucuronide, methyl- $\alpha$-glucopyranoside, sorbitol, cinnamoylglycine, and a range of lipid species) were excluded from entering the candidate biomarker list (Additional file 2: Table S3) because they can also be retrieved in foodstuffs other than mammalian meat. Based on references retrieved in full text or secondary sources, three additional articles were added. As a result, 19 papers were included containing information on 31 putative biomarkers (including ten unknown metabolites) related to red meat intake.

Acylcarnitines, carnitine, 3-dehydrocarnitine, anserine, $\beta$-alanine, 4-hydroxyproline, histidine, ${ }^{13} \mathrm{C} /{ }^{12} \mathrm{C},{ }^{15} \mathrm{~N} /{ }^{14} \mathrm{~N}$, carnosine, creatine, $1-\mathrm{MH}$, and 3-MH have been found to increase after red meat consumption (Additional file 2: Table S3), but these compounds or their precursors are also associated with the overall intake of any meat and they are therefore not necessarily resulting from intake of red meat. The products of cooking of meat mentioned in this review are also unspecific with respect to the type of meat that is cooked. Therefore, these compounds are not suited as red meat-specific biomarkers and have been discussed in the appropriate sections on biomarkers of general, cooked, and processed meat intake.

\section{Compounds potentially specific to red meat intake}

Only three candidate markers, i.e., ferritin, apparent total $\mathrm{N}$-nitroso compounds (ATNCs) (a term that encompasses nitrosothiols, nitrosyl iron, and other N-nitroso compounds (NOCs)), and 1,4-dihydroxynonane mercapturic acid (DHN-MA), were found as potentially red meat specific intake biomarkers. This is because these 
compounds may be formed at a significantly increased level as a consequence of the heme iron content of consumed meat.

Ferritin is a protein that carries and stores iron in the body, and its abundance in serum is thus related to the dietary intake of iron. Serum ferritin is more strongly associated with heme iron than non-heme iron or total iron intake [97]. However, the influence of other dietary constituents on iron absorption and serum ferritin concentration makes it difficult to use this protein as a biomarker for an individual's red meat consumption and associations are not consistent in all studies [98-100]. Serum ferritin, therefore, does not seem to be a useful marker of individual red meat intake.

Heme iron is known to catalyze the formation of nitritederived NOCs associated with the formation of ATNCs. Heme iron also catalyzes formation of lipid peroxidation products (e.g., 4-hydroxy-2-nonenal (4-HNE) [101, 102]. The electrophilic nature of 4-HNE causes its conjugation with glutathione and this adduct is further metabolized into DHN-MA. Several intervention studies confirm the positive association between ATNCs and DHN-MA on the one hand, and heme iron content and/or increasing doses of red meat on the other hand [101, 103-105]. However, validation of DHN-MA as biomarker for red meat intake remains insufficient since only eight male volunteers were included in an intervention study for DHN-MA [105]. Additional information concerning the presence of DHN-MA in a larger and more representative study (e.g., including female test subjects as well) is needed. Furthermore, the DHN-MA precursor, 4-HNE, has previously been detected in foodstuffs other than red meat (e.g., in fish, edible oils, and fried foods [106]), limiting the specificity of DHN-MA as a red meat intake biomarker. For ATNCs, three independent intervention studies have been performed (encompassing a larger group of volunteers, including female test subjects), confirming the link between red meat intake and higher ATNCs levels in stool $[101,103,104]$. However, ATNCs have also been detected in nitrate or nitrite (as precursors of ATNCs) containing foodstuffs, e.g., cured meat (see section on "processed meat"), some cheeses, and beer [107, 108], questioning the use of ATNCs as a qualitative biomarker for red meat consumption. In addition, the analysis of ATNCs has been performed by a method involving thermal release of nitrosamines. In an earlier publication, this heating process has been shown to lead to artefactual formation of nitrosamines while no formation of any simple volatile nitrosamines was detectable [109]. In a study examining the factors affecting ATNCs, all meats, meat iron, and nitrate in the food were directly associated with their formation while vitamin $C$ and total energy intake were inversely associated [110]. While ATNCs apparently associate strongly with red meat intake, the marker still needs validation by another independent and direct analysis of nitrosamines in human feces without the use of thermal sample treatments. In conclusion, there are currently no reliable candidate biomarkers of red meat intake.

\section{Offal meat}

As reported in Additional file 1: Table S2, the search for offal meat biomarkers was restricted by the use of "NOT"-terms to limit the number of irrelevant papers (the search without these NOT-terms yielded a total number of $>100,000$ papers). With the use of the selected NOT-terms, the search for offal meat biomarker papers yielded 647 papers in PubMed and 265 in Web of Science. After combination of the results and elimination of duplicates, the remaining number of papers was 671. Only a single relevant paper [105] includes two putative biomarkers for the intake of blood sausage (and liver paté) in rats and humans: DHN-MA and 8-isoprostaglandin-F2alpha (8-iso-PGF2A), which are both known end products of lipid peroxidation.

Pierre et al. [105] report that urinary levels of 8-isoPGF2A and DHN-MA increased in rats after the consumption of blood sausage. An increased excretion of DHN-MA, but not 8-iso-PGF2A, was subsequently observed in humans [105]. The results of this small-scale human study also show a trend toward an increase in DHN-MA after the consumption of liver paté, although this effect did not appear to be significant; both blood sausage and liver pate contain pre-formed 4-hydroxynonenal, which may have further complicated the interpretation. The authors ascribed the observed increase of 8-iso-PGF2A and DHNMA to the high heme content of blood sausage, corroborating the potential use of the latter as a candidate heme intake biomarker. Consequently, any heme-related BFI would be likely to rise after the consumption of all meat products rich in heme-iron, including red meat, blood products, and liver. Neither 8-iso-PGF2A nor DHN-MA is therefore specifically related to the consumption of offal meat, although the latter may be a lipid peroxidation product more abundant after heme-rich food consumption, as described above. In conclusion, no candidate biomarkers of offal meat intake were identified in the literature search.

\section{Poultry}

Poultry meat intake includes mainly farmed chicken, duck, goose, and turkey; however, wild fowl is expected to have similar markers. The term "white meat" is often used interchangeably to cover either poultry only, or to include poultry as well as fish and shellfish. As already mentioned, some poultry (e.g., duck and fowl) has reddish muscle and this is also the case for several species of larger fish, including tuna. In this review, we have included the intake markers for the different meats with 
their biological class of origin and "white" meats in this section include only poultry.

The literature search performed identified a total of 2311 articles from the three databases. This was reduced to 2055 articles after removal of duplicates. Sixteen articles were identified after screening on the basis of title and abstract. Exclusion criteria for the remaining 2026 articles included effects on physiology; effects on drug metabolism; articles related to antioxidant markers, disease/health markers, or oxidative stress markers; and other articles not relevant to biomarkers of poultry intake by humans, such as animal studies. Full texts of the 16 papers were downloaded and assessed further for exclusion/inclusion criteria. Exclusion criteria at this stage included inappropriate study design, studies on whole diets or on food preparation, and articles not specific to poultry meat intake. Eight articles were retained, focused on poultry products. Additional file 2: Table S3 provides a summary of remaining candidate biomarkers for poultry meat. Only studies on chicken or turkey intake were identified through this search process.

\section{Anserine and methylhistidines}

As previously mentioned, we identified a certain degree of confusion surrounding the nomenclature of $1-\mathrm{MH}$ and 3$\mathrm{MH}$. Based on IUPAC nomenclature, 3-MH and not 1-MH is of interest with respect to poultry consumption [46]. Dietary intake of poultry meat is consistently associated with urinary excretion of 3-MH [44, 111], although a low endogenous source of this compound (3-MH in the correct nomenclature, $1-\mathrm{MH}$ in the article by Giesecke et al.) may also exist in mammals, including humans [56], leading to a low level of background 3-MH excretion in all studies. Excretion of 3-MH in urine $[38,40]$ as well as peak postprandial plasma levels show dose-response within normal levels of chicken intake and plasma 3-MH is still measurable after overnight fasting [46]. The reported levels vary between quantitative studies [40, 46] indicating analytical issues or instability, and inter-laboratory comparisons are therefore needed.

The dipeptide, anserine, was reported to increase in both plasma $\left(C_{\max }=2.72 \pm 1.08 \mu \mathrm{M}\right.$ at $\left.100 \mathrm{~min}\right)$ and urine samples after ingestion of chicken or chicken broth in healthy women $(n=4)$ [37]. Plasma anserine has been associated with poultry intake in a dose-response fashion and high levels were especially associated with selfreported ingestion of turkey in a cross-sectional study from Bavaria [37]. Urinary anserine was particularly associated with chicken intake in high versus non-consumers in a small subset of EPIC participants [38]; however, no information exists on its excretion kinetics.

Guanidinoacetate, a precursor compound in the biosynthesis of creatine found to increase muscle creatine and muscle growth in chicken [112], has been found by NMR analysis in urine as a result of chicken consumption [46].
The compound was found to associate strongly with chicken intake in an intervention study in the UK and this finding was confirmed in a cross-sectional study from Ireland [46]. Guanidinoacetate in urine discriminated intake of chicken from intake of other meat sources and reflected chicken intake in a dose-response fashion. In a dietary study with mixed dairy and meat proteins, including poultry, provided to Danish boys, guanidinoacetate was also observed by NMR analysis [113]. Further validation of the marker is needed from other countries and for other sources of poultry, in particular to determine if the excretion of this metabolite is in any way a consequence of animal feeds [114]. Up until then, this marker seems particularly promising as a unique chicken intake marker.

Several markers were also reported as being associated with cooking methods such as fried and grilled chicken. The putative markers PhIP (2-amino-1-methyl-6-phenylimidazo[4,5-b]pyridine), MeIQx (2-amino-3,8-dimethylimidazol4,5-f quinoxaline), and their metabolites are extensively covered in the next section of this review. Similarly, metabolites related to polycyclic aromatic hydrocarbons (PAHs) detected after consumption of grilled chicken are also reported there. Although levels of these metabolites are increased following consumption of cooked chicken, they are also found in other cooked foods such as fish and beef $[115,116]$, and consequently lack specificity. In conclusion, 3-MH and anserine in urine and plasma should be considered as candidate biomarkers of poultry intake and guanidinoacetate in urine as a highly promising marker of chicken intake. Anserine, in particular, will largely benefit from information on its kinetic profile and dose-response. Overall, additional studies with other poultry meats are needed for further confirmation and validation of all three markers.

\section{Intake biomarkers for heated meat}

Most meat is ingested after cooking; frying, broiling, baking, and grilling are commonly reported as the most popular cooking methods for meat products [117]; however, actual frequencies of different cooking practices are not often reported. In the search for dietary exposure markers for fried, broiled, or grilled meat, 391 unique papers were identified, resulting in 26 selected papers after the screening process and additionally 13 from the secondary search and reference lists (Additional file 1: Figure S1). A total of 39 putative markers for cooked meet have been identified. All compounds belong to the families of heterocyclic aromatic amines (HAAs) and PAHs. One study, based on untargeted metabolomics, reported a series of pyrraline derivatives which were positively associated with the heating process, but the contribution of heated meat could not be separated from those of other cooked foods [118]. Therefore, such compounds were disregarded in the final table. The final 
putative biomarkers are reported in Additional file 2: Table S3.

\section{Heterocyclic aromatic amines}

HAAs may represent specific markers for the intake of intensively cooked meat, as they are produced during hightemperature cooking of foods containing creatinine, creatine, and amino acids, which is the case only for muscle tissue. So far, more than 25 different heterocyclic aromatic amines have been found in roasted and fried meat and fish [119, 120]. Their content depends on the cooking method, but it has been shown that in real-life conditions the most abundant HAAs are PhIP and MeIQx $[120,121]$. Only a small fraction of the HAAs can be found intact in urine, while the rest undergo biotransformation, including phase I metabolism by CYP1A2 and phase II metabolism by acetylation, glucuronidation, and sulphation. As a result, bio-fluids and tissues (e.g., urine, stool, blood, or hair) may contain a complex metabolic pattern reflecting the exposure to specific HAAs [122, 123]. The most reactive metabolites can also covalently bind DNA or proteins, producing adducts, which can be detected in DNA or tissue as well as in urine after DNA-repair or protein degradation [122, 124]. The use of adduct markers is limited due to the low abundance of these compounds and the invasive techniques sometimes required to collect the relevant tissue. Indeed, the majority of these investigations have been conducted on biopsy samples of patients that were obtained during clinical diagnosis of cancer [122]. However, some studies also used blood protein adducts to albumin or erythrocyte globin [124].

Several studies have examined possible markers related to acute HAA exposure in humans after the intake of roasted, fried, or grilled beef, chicken, and/or fish, focusing on the analysis of both free and conjugated HAAs in urine. The most investigated compounds so far are PhIP and MeIQx, which have been quantified before and after enzymatic hydrolysis [121, 125-127] and as their phase I and II metabolites [128-130] (Additional file 2: Table S3). These studies show that PhIP and MeIQx are mainly transformed through hydroxylation followed by conjugation, with glucuronidation as the major urinary excretion pathway in humans. The biotransformation products are completely excreted within $24 \mathrm{~h}$ after single intakes of roasted meat $[128,130]$. After volunteers had consumed identical amounts of charbroiled beef for four consecutive days, the excretion of PhIP in enzymatically hydrolyzed urine decreased almost to baseline levels $48-72 \mathrm{~h}$ later, with a significant correlation between the urinary levels of PhIP and the amount of charbroiled meat consumed [125].

The levels of MeIQx in hydrolyzed urine were higher than those of PhIP $[121,126]$, suggesting a higher degree of direct conjugation of MeIQx, or a relatively greater excretion of metabolized PhIP through feces, as shown by Vanhaecke et al. [131]. When urine was not hydrolyzed, only $\mathrm{PhIP}$ was detected in urine after the intake of a single meal of roasted chicken, whereas unconjugated MeIQx and 4,8-DiMeIQx, which were found in the fried chicken, remained undetected [132]. Although the amounts of HAA metabolites measured in urine were highly associated with the ingested dose of MeIQx and PhIP, high inter- $[116,133]$ and intra-individual [128] variability in metabolism of the HAAs was observed. Notably, differences between "fast" and "slow" excretors as well as between men and women were observed $[128,130]$.

In observational studies, MeIQx has been found to be a more reliable urinary marker to assess consumption of highly cooked or processed meat. The urinary levels of MeIQx [134] and PhIP [135] have been compared among black, Asian (Chinese or Japanese), and white men, and correlated with the results of a food frequency questionnaire. MeIQx levels were positively associated with the intake frequency of bacon, pork/ ham, and sausage/luncheon meats [134] while urinary PhIP was not associated with intake frequencies of any cooked meat, suggesting that self-administered dietary questionnaires may not properly describe cooked meat preferences, frequency of intake, and cooking methods [135], or alternatively that unknown sources of PhIP affect total excretion.

Further markers related with dietary HAA exposure are the PhIP hydroxylation metabolites. 4'-OH-PhIP (2-amino1-methyl-6-(4'-hydroxyphenyl)imidazo[4,5-b]pyridine) has been proposed as detoxification product, derived from the oxidation by several CYPs of the aromatic ring system. The metabolite, 5-OH-PhIP (2-amino-1-methyl-6-(5-hydroxy)phenylimidazo[4,5- $b]$ pyridine), has been suggested as a marker of activation of PhIP [136] since it represents a rearranged product of $\mathrm{N}$-hydroxylation of the exocyclic amino group by cytochrome P450 (CYP1A2). In several studies [121, 132, 137], 4'-OH-PhIP was found to be the most abundant urinary hydroxylated metabolite after the consumption of cooked chicken. This compound is already abundantly present in the heated chicken meat and only a small amount (11\% after enzymatic hydrolysis and $0.66-1.3 \%$ in untreated urine samples) is derived from human PhIP metabolism $[132,137]$. This suggests that $4^{\prime}-\mathrm{OH}-\mathrm{PhIP}$ may represent a good marker for heated meat intake, although studies showing its presence and kinetics of formation in other meats than chicken are needed in order to validate its plausibility as a general marker of any fried or grilled meat.

High inter-individual differences were observed for fecal excretion with no differences in the percentage of the PhIP dose excreted [131]. Vanhaecke et al. [131] examined the urinary and fecal excretion in humans of a newly identified microbial PhIP metabolite, PhIP-M1 (7-hydroxy-5-methyl-3-phenyl-6,7, 8,9-tetrahydropyrido[ $\left[3^{\prime}, 2^{\prime}: 4,5\right]$ imidazo[1,2-a]pyrimidin-5-ium chloride) after the consumption of cooked chicken, containing a known amount of PhIP. Urinary PhIP-M1 increased over time with a peak between 48 and $72 \mathrm{~h}$, showing that microbial metabolism appears later in the excretion profile of 
body fluids compared to the parent compound. The percentage of recovered PhIP-M1 was quite high in feces, with high variability among subjects, probably associated with differences in diet, digestion, metabolism, and microbial composition. There was no difference in the percentage of the PhIP dose excreted as PhIP-M1, indicating apparent doseresponse. In conclusion, this additional microbial PhIP metabolite, PhIP-M1, adds to the complex metabolism of PhIP and may further complicate the use of any PhIP metabolite for monitoring exposures to heated meats.

Other compounds besides PhIP and MeIQx have been identified in urine after the intake of roasted meat (Additional file 2: Table S3). These include $9 H$-pyrido[3, 4-b]indole (norharman) [132, 138], IQ (2-Amino-3methylimidazo[4,5-f] quinoline), Trp-P-2 (3-amino-1methyl-5H-pyrido[3,4-b]indole), Trp-P-1 (3-amino-1, 4-dimethyl-5H-pyrido[3,4- $b$ ]indole), and $\mathrm{A} \alpha \mathrm{C}$ (2amino-9H-pyrido[2,3- $b$ ]indole) and harman [138]. However, these molecules were found to be less reliable as markers of exposure to heated meat, when compared to PhIP and MeIQx. Their presence in body fluids or tissue may not be entirely due to meat consumption, but also to smoking habits [139-141], endogenous production, and the consumption of other foods derived from heating or fermentation, such as alcoholic drinks and coffee [142].

Markers related to the intake of high-intensity cooked meat have been investigated also in human hair, providing an average of the exposure over a period of several months [143-150]. Analysis of hair samples showing doseresponse, a known dependence on melanin levels and independence of hair dying, and CYP1A2 phenotype, has been shown for measurement of PhIP after fried beef intake for 3-4 weeks [143-145]. The lack of dependence on metabolism is in accordance with findings showing that CYP1A2 phenotype over a 20-fold activity span is a poor predictor of HAA metabolite excretion in urine [150]. Turesky et al. [149] examined the levels of PhIP accumulating in the hair closest to the scalp of 44 volunteers after a 4-week semicontrolled diet of cooked meat containing known quantities of PhIP. The study showed a good correlation between the level of PhIP in hair and the dose of PhIP ingested. Kobayashi et al. [144] compared PhIP levels in the hair of seven volunteers with the results from dietary records over 28 consecutive days. PhIP levels in hair were found to be highly associated with the grilled/stir-fried meat intake but not with the grilled/stir-fried fish intake. The marker, therefore, seems to be useful in very different populations in terms of food culture and genetics. No other HAAs can be measured in hair and no, or only marginal, PhIP could be measured in vegetarians. These studies point to PhIP in hair as a promising biomarker for assessment of average intakes of heated terrestrial meats but further validation studies are needed to assess stability of the samples and influence of hair structures.
In conclusion, the studies reported so far identified and quantified specific carcinogenic HAAs in urine, feces, and hair after consumption of well-done, heated meat, showing that there is a significant correlation between the intake and the excretion of these compounds. In particular, the most promising exposure markers seem to be total PhIP and MeIQx in urine and PhIP in hair. The complexity of absorption, metabolism, and distribution of these compounds leads to very high interand intra-individual variation for some of the blood and urine metabolites and these compounds may therefore only work reliably as markers for comparison of groups rather than individuals. PhIP in hair seems a consistent candidate biomarker to estimate intakes of fried or grilled terrestrial meat.

\section{Polycyclic aromatic hydrocarbons}

PAHs are mainly produced from the incomplete combustion of organic matter. Therefore, the main human exposure sources are associated with environmental factors, such as tobacco smokes, emission of vehicles, and occupational exposure [151]. Anyway, it has been observed that in nonsmoking subjects without occupational exposure, the main source of PAHs is represented by food [152, 153]. PAHs are present both in uncooked and cooked food. In the former, these compounds may originate from environmental contamination from soil, air, or water [154]. Cooked food, particularly meat grilled directly over an open flame, grilled over charcoal, or smoked, may contain measurable amounts of PAHs arising from fat pyrolysis or from the adsorption onto foods of PAHs emitted from the combustion process [155]. The most abundant PAHs reported in barbequed or grilled meat were naphthalene (NAP), phenanthrene (PHE), fluorine, and pyrene [152, 156].

Urinary 1-hydroxypyrene (1-OHP) and 1-hydroxypyreneO-glucuronide (1-OHPG), two metabolites of pyrene, are considered the most relevant biomarkers for assessing individual exposure to PAHs [157-160]. Even though they have been observed to increase following the consumption of barbecued meat, a very high inter-individual variability has been observed, suggesting that these metabolites may not be suitable markers for individual assessment of the intakes of grilled, broiled, or roasted meat [158-160]. Furthermore, it has been reported that the main source of urinary 1-OHPG is cigarette smoke, while car vehicle exhaust contributed almost to the same extent as the diet in non-smoking subjects [161]. A considerable amount of 1-OHPG excretion is also attributed in populations using mate tea while fried or grilled meat was not significantly associated with 1-OHPG [162]. Cooking methods (baking bread at home and the intensity of food frying), passive smoking, and genetic polymorphisms affecting hydroxylation and conjugation, additionally highly contributed to the excretion of 1-OHPG [163]. Therefore, the background levels for 1-OHPG would be too variable in a real 
exposure setting and the contribution of dietary intake of high-intensity heated meat could not be distinguished from environmental factors, even in subjects who never smoked.

Further studies identified monohydroxylated metabolites of NAP and PHE in urine as possible markers of shortterm dietary intake of PAHs after consumption of fried beef and chicken, particularly 2-hydroxy-NAP and 1-, 2-, 3-, 4-, and 9-hydroxy-PHE $[152,156]$. These metabolites demonstrated a high correlation with the parent compound present in food in a highly controlled setting where the environmental confounders were minimized. Even though these metabolites show a higher robustness in relation to the other environmental factors, the existence of other background source acting as potential confounders cannot be overruled. NAP and PHE are well-known environmental contaminants causing aquatic toxicity and they are therefore likely to show environmental bias similar to other PAH related markers [164].

In conclusion, none of the markers of PAH intake are currently promising markers of charcoal-grilled meat intake.

\section{Processed meat}

The search for papers on processed (not including heat processing) meat biomarkers delivered 2522 papers via PubMed and 2560 papers from Web of Science. This rendered 3772 unique papers, of which 38 were included after screening of the titles and abstracts, whereas only six relevant papers remained after assessment of the full text. Exclusion was based on a lack of focus on the assessment of dietary intake in general, and processed meat intake in particular. Four additional papers were retrieved after the secondary web search. In total, 12 relevant papers were found, reporting putative markers such as MeIQx, 1-OHP, thiobarbituric acid reactive substances (TBARS), ATNCs, nitrosoproline, glycerophosphatidylcholine (PC) C38:4 (PC38:4), acetylcarnitine, carnitine, and lathosterol. Out of these, MeIQx and 1-OHP are also markers of grilled and fried meat; ATCN and DHN-MA are potential markers of heme-containing foods; carnitine and acyl-carnitines are candidate markers of meat intake in general; and lathosterol, TBARS, and PC38:4 are unspecific to processed meat intake. These compounds were therefore disregarded as candidate markers of processed meat. However, they may still be useful as part of combined markers for processed meat intake assessment. This leaves $\mathrm{N}$-nitrosoproline as the only candidate biomarker for this specific meat subgroup.

\section{$\mathrm{N}$-nitrosoproline}

Decades ago, Stich and co-workers [165] investigated the interfering role of the consumption of nitrite-preserved meats on urinary $\mathrm{N}$-nitrosoproline concentrations. The results demonstrated that $\mathrm{N}$-nitrosoproline increased significantly after the consumption of, e.g., tinned ham, pork luncheon meat, and different types of sausages, although
$\mathrm{N}$-nitrosoproline can also be formed endogenously in the gastro-intestinal tract. The authors were able to directly link $\mathrm{N}$-nitrosoproline levels in different processed meat types to higher levels of $\mathrm{N}$-nitrosoproline in human urine. Moreover, endogenous $\mathrm{N}$-nitrosoproline formation appeared to be negligible in comparison with the levels formed after intake of cured meats. N-nitrosoproline therefore demonstrated potential to serve as a candidate quantitative biomarker for the intake of nitrite-preserved meat. Later on, other investigations showed that nitrite-preserved meat is not the sole or even the main potential contributor to elevated urinary $\mathrm{N}$ nitrosoproline $[166,167]$ since tobacco use is another important source, eliminating $\mathrm{N}$-nitrosoproline as a robust, specific biomarker for nitrite-preserved meat intake. However, other sources may be identified with other biomarkers and combinations of markers may therefore be developed to assess intake of cured meat besides other environmental or food sources. Further studies of N-nitrosoproline in complex exposure scenarios are therefore warranted.

\section{Unidentified potential markers of processed meats}

In studies by Playdon et al. (2016) and Cheung et al. (2017), the occurrence of five unidentified compounds was associated with the intake of processed meat [38, 62]. Additional research is required to identify these molecules and assess their relevance as markers of processed meat intake.

In conclusion, only a limited amount of original research papers document the search for a processed meat biomarker. More importantly, up until now, none were able to identify a biomarker that is specific for processed meat intake.

\section{Biomarkers of fish, fish oil, and other seafood intake}

Three parallel searches of the scientific literature have been carried out for markers of aquatic meats and oils: one search addressed the identification of markers related to fish and fish oil intake, another on seafood, and a third search was focused on naturally occurring environmental toxicants related to seafood consumption (Additional file 1: Table S2).

A number of 1340 unique articles were identified for the search on fish and fish oil biomarkers of which 1115 articles were discarded in the first screening based on title and abstract. The main reasons for exclusion were effects on physiology, bone health and calcium metabolism, macular degeneration, effects on drug metabolism, as well as studies on effects in fish. Out of the 225 papers included in the second screening for which the full-text was assessed, 55 were kept and are included in Additional file 2: Table S3 together with 19 papers identified from the secondary searches. Papers that were discarded dealt with irrelevant diets (pure n-3 fatty acids, not fish oil), arachidonic acids, seal, astaxanthin from microbes, etc.), outcomes (effects on cholesterol levels, 1-carbon metabolism, immunology, fatty acid levels in breast 
milk, neurological development of newborns, urinary iodine excretion), or were excluded due to poorly reported methodology or inappropriate study designs for BFI discovery. One proteomics study unrelated to biomarker discovery and four studies for which the access links were unavailable were also excluded. Several additional studies arose from the literature search within the general meat section, while one crosssectional and one intervention study were found through the targeted searches on TMAO and CMPF (3-carboxy-4-methyl-5-propyl-2-furanpropionic acid), adding a total of 15 papers to the final list $(n=86)$. Among the papers included in Additional file 2: Table S3, 45 are relevant for fish consumption, 34 for fish oil supplementation, and five investigate both fish and fish oil mixed intakes.

The literature search for shellfish resulted in $443 \mathrm{pa}$ pers, of which 33 were kept after screening abstracts and titles. The reasons for exclusion were investigation of contaminants in seafood and their effects on health, effects on physiology of aquatic food consumption, and food allergies. After accurate examination of the text, the remaining papers included 19 mentioning shellfish, 13 on mixed intakes together with fish, and six only on shellfish, see Additional file 1: Figure S1.

For the search on natural environmental contaminants associated with the intake of aquatic meats, only arsenocompounds have been considered. The literature search resulted in 100 papers, which were reduced to 14 after screening abstracts and titles. Generic markers for inorganic arsenic exposure were excluded. Other reasons for exclusion within this category were irrelevant dietary intervention (e.g., arseno-sugar and cod liver) and a lack of firm conclusions in associating specific foods to the markers of interest. Only eight papers measuring organoarsenic species in body fluids and tissues in relation to fish, mixed fish, or shellfish intakes were included and these papers are therefore listed under the fish, or mixed fish and shellfish categories.

In Additional file 2: Table S3, the studies on intake biomarkers of fish, fish oil, shellfish, and mixed exposures of these are listed in separate sections; however, due to the large overlap in the compounds identified as markers, the foods are all discussed under one heading here and subdivided by chemical class.

Several biomarkers have been found to be associated with intake of fish and shellfish as reported in Additional file 2: Table S3. The majority of the studies concern consumption of marine foods and derived products aimed to increase the levels of omega-3 (n-3) long-chain polyunsaturated fatty acids (n-3 LCPUFAs) in biofluids and tissues, while more recently suggested markers include furan fatty acids, e.g., CMPF, TMAO, 1,2,3,4-tetrahydro- $\beta$-carboline-3-carboxylic acid (THCC), cetoleic acid, astaxanthin, and organoarsenic compounds, such as arsenobetaine. Fish and shellfish are reasonably high in the trophic chain and tend to accumulate compounds present in their local pray or foods. This accumulation depends on the biotope, e.g., marine or fresh waters, geographical differences, and also on the fat content of the fish or shellfish. A high abundance of LCPUFAs, including docosahexaenoic acid (DHA), exists in marine plankton [168] while freshwater microalgae are generally forming other fats and only little eicosapentaenoic acid (EPA) [169]. Most fatty acids and xanthophylls are deposited in fats and accumulate more in fatty fish while TMAO is produced in some species to regulate osmotic pressure in deeper marine waters. Biomarkers of fish and shellfish may therefore depend on the local environments where these foods come from.

\section{n-3 LCPUFAs}

The n-3 LCPUFAs, EPA and DHA, as well as their intermediate product, docosapentaenoic acid (DPA), are high in marine fatty fish and in cod liver. Two to 20 times lower levels are found in most other aquatic meats, including shellfish, lean fish, some freshwater fish, and farmed fish from coastal waters or ponds, while terrestrial meat are 20100 times lower, depending on their feed [170]. The n-3 LCPUFAs in blood are well-established fish-related biomarkers, known for their high specificity to fish and shellfish. In particular, fatty fish such as salmon, tuna, herring, and mackerel are affecting blood n-3 LCPUFAs [171]. Although eggs and meats originating from livestock fed with diets high in n-3 may also provide some quantities of EPA and DHA (e.g., through their higher consumption rates in many populations), marine and freshwater foods represent by far the main dietary source of n-3 LCPUFAs $[171,172]$. LCPUFAs can additionally be synthesized in humans by converting $\alpha$-linolenic acid (ALA) through a welldocumented series of elongations and desaturations [173]. However, the conversion rate of ALA from plant sources is limited [174] and should therefore not interfere with the net content provided by marine sources.

EPA and DHA have often been used to assess compliance to fish and fish oil intake in intervention studies [175-179], and have been extensively associated with self-reported fish intake, measured either through 24-h dietary recalls or FFQs in a wide range of cohort and cross-sectional studies carried out in different populations [45, 180-183]. Furthermore, EPA and DHA have been widely used to validate proposed FFQs for assessment of fish intake [83, 184-187]. DHA, DPA, and EPA were also shown to increase in a $12 \mathrm{w}$ parallel RCT in adolescents comparing fish meals (herring, salmon, mackerel) with meat (chicken, turkey, beef, lamb) and cheese [188].

Blood is the most extensively explored matrix for measuring PUFAs; however, blood components differ in their metabolism and incorporation of LCPUFAs [189], thereby greatly influencing the duration of consumption 
reflected and the selection of the adequate blood fraction to match the intended outcome.

Plasma and serum have been suggested to reflect shorterterm intakes, whereas erythrocyte membranes and adipose tissue are more reflective of long-term (habitual) consumption of fish [190]. In serum, EPA, DHA, and total n-3 fatty acids increase within $4 \mathrm{~h}$ after intake [191], whereas their levels in cholesteryl esters (CE) and red blood cells (RBC) were only apparent after 3 days of supplementation. Incorporation of EPA and DHA was also shown in PC after 1-2 weeks, whereas levels in platelets were affected after 3-4 weeks and 1-2 months, respectively [192]. The incorporation of DHA was further reported to be slower than that of EPA in CE, RBC membranes, and the adipose tissue of healthy adults [193].

Plasma phospholipids act as transporters and are therefore a more transient compartment than RBC membranes [190, 194], with equilibrium reached after 1 and 6 months, respectively, at fixed dietary intakes [195]. The clearance of EPA and DHA from plasma phospholipids was also shown to occur more rapidly than from RBC [196], emphasizing on the ability of plasma phospholipids to adequately reflect only short-term changes in intake (weeks). Esterified LCPUFAs in whole blood were associated as strongly as LCPUFAs in erythrocytes in a fish oil intervention with $0.25-1 \mathrm{~g} /$ day of EPA and DHA over 4 weeks [197], and were shown to have similar kinetics within RBCs and in plasma, while EPA has faster kinetics than DHA [198]. The sum of EPA + DHA in plasma PCs was suggested as a suitable biomarker for recent EPA + DHA intake, while platelet and mononuclear cell EPA + DHA may reflect habitual intakes, showing reasonable dose-response relationship [192]. PC-EPA was also reported in an untargeted metabolomics investigation with fish oil [199] as well as in a cross-sectional association with aquatic meats [200], both measured in serum. Fish studies targeting PC-EPA and PC-EPA + PC-DHA may thus potentially shed light upon more accurate assessment of short-term fish intake.

Adipose tissue is less sensitive to changes in dietary n3 PUFA than other lipid pools, thus fatty acids content in adipose tissue reflects only longer-term intake of fish oil [193, 201].

In studies where similar content of EPA and DHA was provided as either fish oil capsules or fatty fish, differentiation by plasma markers between these intakes is not possible $[189,202]$, nor is it possible to reflect different composition of the fish and fish oil [203].

Only one intervention study investigated the potential dose-response relationship between LCPUFAs after fish consumption [204]. EPA measured in plasma phospholipids showed dose-response at doses of $180 \mathrm{~g}$ and $270 \mathrm{~g}$ of salmon consumed twice a week for 4 weeks, but no significant difference from control was observed at $90 \mathrm{~g}$ of salmon. In contrast, DHA seemed to reach a plateau in this study even after
$90 \mathrm{~g}$ salmon consumed, without a subsequent increase at higher doses [204]. When added together, EPA + DHA showed a dose-response starting with the lowest level of fish intake [204]. Other studies that investigated dose-response only used fish oils. In blood samples, EPA and DHA displayed dose-response in some studies with fish oil [193, 205-207, 209, 210]. Ulven and colleagues compared the efficacy of fish or krill (Euphausia superba, a crustacean) oils on n-3 LCPUFA contents in fasting plasma samples of healthy volunteers. Despite lower n-3 contents in krill oil, effects comparable to fish oil were observed for EPA, DPA, and DHA, suggesting that bioavailability of n-3 PUFA from krill oil is equal or even more efficient than that of fish oil [211]. The different efficiency may be attributed to different n-3 containing fats in krill oil, mainly phospholipids, compared with fish oil, mainly triglycerides. In a 14-day dose-response study, Hudson et al. have shown that incorporation of EPA, DPA, and DHA in PC shows doseresponse in the range from 0.3-4.5 g EPA + DHA/day so that contents in $\mathrm{PC}$ is a sensitive and dynamic measure of recent LCPUFA intakes [212].

The use of DHA and EPA as BFIs for fish are likely to reflect mainly marine fish and should therefore be validated separately for different populations, as always recommended for BFIs [24]. In experimental studies, lean fish only affect n3 LCPUFAs to a very limited extent [213] while these markers in observational studies have less power to discriminate lean and fatty fish intakes [180, 214-216]. Shellfish have a composition similar to lean fish but most observational studies on EPA and DHA did not differentiate between fish and other shellfish [184, 216, 217]. When shellfish intake was evaluated separately from non-fried fish intake in 900 participants from the Multi-Ethnic Study of Atherosclerosis (MESA), no association was observed between the concentrations of EPA and DHA in plasma phospholipids and shellfish intake [218]. When looking into intakes of more specific shellfish such as oysters, clams, crabs, squid, mussels, and shrimp in normolipidemic men, all shellfish increased n-3 PUFAs in fasting plasma and erythrocytes, except for clams that only elevated them in plasma, and shrimps that only showed an effect on RBC [219]. The plasma levels increased five times more than those in RBC, confirming that the turnover rate in different compartments is affected similarly by fish and shellfish. Tropical shellfish, unlike those from temperate waters, are a source of both n-3 and n-6 PUFAs, as shown by increased plasma levels of arachidonic acid reported together with the regular EPA, DPA, and DHA [220]; this underlines once again that BFIs should always be validated for each specific population taking their food sources into consideration.

\section{Ratios of $n-3 / n-6$ fatty acids}

Fish intake negatively affects the plasma levels of certain omega-6 PUFAs. Linoleic acid (LA), gamma-linolenic 
acid (GLA), dihomogammalinolenic acid (DGLA), and arachidonic acid (AA), all n- 6 PUFAs, have often been reported in studies on fish intake, but are of limited interest as fish intake biomarkers since they do not reflect fish intake per se but rather a change in their equilibrium with the n-3 FAs. Consequently, FA ratios such as $n-3 / n-6, n-6 / n-3$, the omega-3 index, and EPA/AA may be of potential relevance for health outcomes related to fish consumption, but are not robust as BFIs, as they are highly influenced by other food sources, although they may be relevant in some specific studies $[204,214,221-224]$. The same applies to the reported sum of $n-3$ and sum of n- 6 PUFAs, measured in a handful of studies reported in Additional file 2: Table S3 $[176,191,201,204,213,214,225-233]$, as they can easily be affected by the intake of other food groups than aquatic or terrestrial meat, such as vegetable oils and nuts.

In conclusion, n-3 LCPUFA in different blood compartments seem to be valid biomarkers of fatty fish intake and also of total fish, fish oil, and shellfish intake in populations consuming a mixed diet. The ratios, EPA/ AA, (EPA + DHA)/AA, and (n-6)/LCPUFA may only be useful in controlled studies comparing fish and meat since they are too sensitive to other n-6 FA sources to be useful as general fish intake biomarkers.

\section{Furan fatty acids}

In addition to DHA and EPA, furan fatty acids have been recently suggested as biomarkers for fish/fish oil intake. CMPF was identified as a biomarker for fish consumption in fasting plasma, as its level was associated with dietary fish intake after a 12-week intervention trial with a Nordic diet [234]. In addition, another dietary intervention study has reported increased furan fatty acids after increased protein intake from fish and meat, including circulating levels of CMPF following consumption of a Mediterranean-type diet [235]. Plasma, serum, and urine CMPF has been observed also in free living populations across the Northern Hemisphere, where levels were associated with habitual dietary intake of fish and shellfish in several populations in Europe, China, and the USA [62, 236, 237]. Plasma as well as urine CMPF has also been observed to increase in hyperlipidemic patients after $600 \mathrm{mg} /$ day of fish oil for 4 weeks and levels were still increased after 4 weeks' wash-out [238]. Besides CMPF, four other compounds putatively identified as furan fatty acid metabolites were increased in blood after a diet high in fish [235, 239].

\section{Trimethylamine-N-oxide}

TMAO is a naturally occurring metabolite abundant in certain fish or generated from nutritional compounds including choline and carnitine by a microbiota mediated conversion of betaines to trimethylamine and further oxidation by hepatic flavin monooxygenases to TMAO [240, 241]. Several studies have shown increased urinary excretion of TMAO related to consumption of fish: a high level of TMAO in morning urine was associated with intake of fish containing PUFA and n-3 PUFA on the previous day [242]. Increased urinary TMAO was also observed within $4.5 \mathrm{~h}$ after a salmon-containing breakfast [47]. In an intervention with fish or meat dishes, urinary TMAO increased substantially after fish intake but not after standardized meals containing chicken, red meat, or processed meat [38]. In another crossover meal study with three fish dishes, three meat dishes and three lacto-ovo vegetarian dishes, TMAO increased in urine only after fish intake, peaking at $3 \mathrm{~h}$ [93]. TMAO in urine seems to show good specificity as a potential biomarker of fish and shellfish intake, because only fish and other aquatic meat products contributed to elevated urinary excretion of trimethylamine and TMAO, among 46 investigated food groups [240]. TMAO levels in plasma showed a dose-response with increased intakes [38], but also increased postprandially after intake of a fish meal, thus proving that the TMAO present in fish can be readily absorbed without the involvement of gut microbiota, while TMAO is elevated much later when metabolic precursors in meat are converted to TMAO by the gut-host co-metabolism [94]. TMAO is particularly high in sharks, skates, cods, and other fish diving in temperate or cold sea waters, serving as a muscle osmolyte, and it is also found in crustaceans from the same biotopes [243]. It may therefore be a better biomarker for intake of this group of fish and shellfish, e.g., from the North Atlantic Ocean. Plasma TMAO has recently evoked attention as potential risk factor for cardiovascular diseases [244]. However, this risk seems to be partially related to reduced kidney function [245] and also depends on the composition of the gut microbiota [94]. Therefore, the potential implication of TMAO from aquatic meat in disease development still needs scrutiny. In conclusion, TMAO in urine is a candidate short-term biomarker of intake for fish and shellfish from deeper water layers, at least in the North Atlantic Ocean, while studies of fish and shellfish from most other biotopes are still missing.

\section{1,2,3,4-Tetrahydro- $\beta$-carboline-3-carboxylic acid}

In a randomized, single-blinded, crossover meal trial, metabolomics analysis was used to investigate plasma and urinary excretion following consumption of cod protein compared to other protein sources, such as whey, casein, or gluten. The tryptophan-derivative THCC was elevated in plasma and urine after cod protein intake [95]. Further studies to evaluate its specificity are needed, given the potential presence of THCC at considerable levels in cocoa 
products and in some fruit juices, jams, and fermented food products [246]. THCC is therefore not considered further as a fish intake biomarker.

\section{Amino acids}

Increased urinary excretion of taurine and 3-methylhistidine have been observed after fish and shellfish intake but have also been associated with animal protein consumption [247]. Though being less specific than TMAO, plasma levels of taurine, methylhistidines, and N6,N6,N6-trimethyl-lysine were also elevated following the consumption of cod protein [95]. Anserine, 1- and 3-methylhistidine were also reported in urine after a smoked salmon-containing breakfast [47]. As methylhistidines and taurine may be derived from several different sources of animal muscle, they are not specific to fish or shellfish and have therefore been discussed in the section on general markers of meat intake. However, in combined biomarker approaches, they may serve to distinguish between intake of fish (as part of "white meat") from intake of mammalian red meats but this approach will need additional study.

\section{Other lipids}

Other lipids have been suggested as possible biomarkers of fish intake. In particular, a lipidomics investigation from O'Gorman et al. [248] demonstrated that a combination of specific lipid patterns had a reasonably good ability to discriminate between low and high dietary fish intake (AUC $=0.76$ ) in 34 volunteers from the Metabolic Challenge Study (MECHE), with lysophosphatidylethanolamine (LPE) acyl C18:2 (LPE (18:2)) and phosphatidylethanolamine diacyl C38:4 (PE (C38:4)) identified as potential biomarkers in serum. Since C18:2 (LA) is an (n-6) fatty acids unrelated to fish intake, the association may be caused by confounding with, e.g., oils used for frying the fish. In another randomized controlled trial, a fatty fish-based diet produced an increase in the levels of lysoPC (20:5) and LPEs (20:5) and (20:6), which are very likely derived directly from the fatty fish [234]. Plasma phospholipid-LCPUFAs may serve as BFIs for shorter term or recent fatty fish intake in analogy with free LCPUFAs as already discussed.

In a study on plasma lipids in overweight men following meals with baked herring or baked beef, cetoleic acid (22: $1)$ increased after herring intake, while 2 -aminoadipic acid, leucine, $\beta$-alanine, and 4-hydroxyproline reflected beef intake [57]. Additional studies of cetoleic acid as potentially specific to intake of herring or of fish in general is needed for confirmation.

\section{Astaxanthin}

Astaxanthin is a natural lipophilic compound, which belongs to the xanthophylls and its bioavailability is enhanced by dietary lipids [249]. While it is found in a variety of aquatic organisms, including algae, krill, crayfish, salmon, salmon roe, shrimp, and crab, particularly high amounts are found in the flesh of salmon and in the carapace of crustaceans [250,251]. No studies exist to evaluate the main food sources of plasma astaxanthin in different populations but in the Western World, salmon and shellfish are likely to be the major sources. Astaxanthin cannot be synthesized by animals or humans, but it is essential for the growth of Atlantic salmon alevins [252] and its presence therefore indicates accumulation from food. Astaxanthin isomers, preferentially absorbed and concentrated into VLDL in humans, contain cis-double bonds, whereas the all-trans astaxanthin is most accumulated in salmonoids [253, 254]. The biokinetics of astaxanthin have only been studied after supplementation. The maximal concentration of astaxanthin in serum is found at $12 \mathrm{~h}$ reaching $\mathrm{C}_{\max }$ of $50-280 \mu \mathrm{g} / \mathrm{L}$ after intake of doses of 10 $100 \mathrm{mg}$ given as supplements and is then declining with a half-life of around 12-21 h [253-255]. Accumulation of astaxanthin has been indicated from doses of 1-3 mg over 4-12 weeks showing dose- and time-dependent increases up to final levels of $11 \mu \mathrm{g} / \mathrm{L}$ after $1 \mathrm{mg} /$ day and $35 \mu \mathrm{g} / \mathrm{L}$ after $3 \mathrm{mg} /$ day at 12 weeks [256]. Daily doses of $4 \mathrm{mg}$ astaxanthin over 3 months were found to provide final plasma levels of around $30 \mu \mathrm{g} / \mathrm{L}$ in reasonable agreement with other data [257]. Feeding of hens with algae, specific yeasts, shellfish waste, or even synthetic astaxanthin has been shown to increase its presence in egg yolk, providing the basis for commercial "golden" eggs [258]. Other food sources in the human diet besides supplements are food colorants, e.g., E161. However, they are likely to be minor in populations ingesting aquatic meats. Background levels, presumably from aquatic meat intake, in middle-aged Japanese males were found to be 0.1-0.5 $\mu \mathrm{g} / \mathrm{L}$ while background levels in Norwegian, German, or Dutch men and in Korean women were reported as non-detectable [254, 256, 259-261]. In groups of English and Russian subjects abstaining from aquatic meat for 10 days, levels were $0.25 \mu \mathrm{g} / \mathrm{L}$ [262]. In a study with 28 German men, recruited to consume $250 \mathrm{~g}$ wild or aqua-cultured salmon providing $5 \mu \mathrm{g}$ astaxanthin/g salmon flesh in a randomized and double-blind trial over 4 weeks, plasma astaxanthin concentrations of around $25 \mu \mathrm{g} / \mathrm{L}$ were detected [261]. The bioavailability from salmon seems therefore comparable to or higher than what was observed with the supplements.

In conclusion, astaxanthin may not be specific to fish and shellfish but in populations with low intakes of other sources of astaxanthin, it can be considered a candidate biomarker of these foods. Since there is good concordance between plasma levels measured in different laboratories, astaxanthin is likely to have good reproducibility. However, inter-individual response to the same dose of astaxanthin was quite large in some of the studies $[256,261]$. 


\section{Organoarsenic compounds}

It has been observed that many persistent organic pollutants (e.g., dioxin and organochlorine pesticides) and inorganic pollutants (e.g., mercury) are highly associated with fish consumption [263]. However, the presence of such compounds and elements in several other food matrices, as well as their differential distribution worldwide, create highly variable background levels, which do not allow the use of such compounds or their metabolites as reliable fish intake biomarkers [264]. Thus, for the search on environmental contaminants associated with the intake of aquatic meat, only arseno-compounds have been considered, given their high specificity to marine foods.

Due to the bioaccumulation of arseno-compounds along the aquatic food chain, fish and shellfish contain significant amounts of arsenic (As). In marine organisms, inorganic As present in ocean water is transformed into unique organic compounds by methylation or esterification with sugar (arsenosugars), lipid (arsenolipids), betaine (arsenobetaine, AsB), or choline (arsenocholine) [265]. AsB and methylated As (dimethylarsinate or DMAs, monomethylarsonate or MMAs) constitute the biggest proportion of As in fish and shellfish, while inorganic As(III) and As(V) are the least abundant metabolites [266]. Urinary profiles of As metabolites thus showed a positive dose-response relationship with recent fish or shellfish intake, despite differences based on species [267] and preparation method, since the watersoluble As metabolites are easily leaking from the meat when boiled [268].

Urinary excretion of DMAs 13-24 h after aquatic meat intake $[266,269]$ shows that As methylation is one of the As detoxification pathways in the human body $[267,268]$. Urinary level of DMAs can thus also reflect As exposure from any other sources, such as seaweed [270], rice, mushroom [271], drinking water [269], or inhalation [272]. Consequently, DMAs and MMAs have low specificity as potential biomarkers of fish intake and are not considered further here.

Another major As metabolite, AsB, was rapidly excreted after fish and shellfish intake [95, 266, 268], showing limited biotransformation and accumulation. Formation of AsB from other As metabolites takes place in humans and AsB also influences urinary DMAs excretion $[267,269]$. The extent of these processes may be limited since AsB was not associated with As content in drinking water [269] or inhaled air [272], and was only weakly associated with rice intake [269]. In addition, urinary AsB is less susceptible to inter-individual variation than other organoarsenic species, especially when expressed as concentration rather than as $\mu \mathrm{g} / \mathrm{g}$ creatinine [266]. Adjustment for AsB or stratified analysis in some subjects with undetectable urinary AsB nullified the association between aquatic meat intake and urinary concentrations of total As or DMAs [270]. This finding supports the specificity of AsB as a biomarker of total fish and shellfish intake and AsB seems to be a promising candidate biomarker for these foods.

The most commonly investigated biospecimen for AsB quantification is urine $[266,269,270]$. We only found one intervention study by Stanstrup and colleagues [95] that examined the biomarkers of acute fish intake in both plasma and urine samples, however without comparing the performance of the two. We did not find any article discussing the measurement of plasma AsB as a potential indicator of habitual fish intake. Hence, current evidence support the usage of plasma AsB to reflect only recent intakes, and urinary AsB as an indicator of both recent (within 3 days) [269] and habitual fish and shellfish intake [270]. The kinetics of AsB varied across intervention studies, peaking in plasma within $4-14 \mathrm{~h}$ and returning to baseline after $8-50 \mathrm{~h}$ for intakes of cod [95, 267] and for urinary excretion after intake of crabs, wolfish, and lemon soles [272]. An observational study showed an association between urinary AsB and fish and shellfish intake within the last $72 \mathrm{~h}$ [266]. Association between urinary AsB and habitual aquatic meat consumption in the past year [270] was also observed at the group level but suggests no retention or tissue accumulation of AsB. Furthermore, as a natural, widespread water pollutant metabolite, AsB levels in fish or shellfish may vary across species and location [267]. Current evidence indicates that AsB may serve as a promising qualitative biomarker of fish and shellfish intake but its use for quantitative purposes needs further investigation. Studies so far were conducted in Caucasian populations in Europe [95, 266, 267, 272] and the US [270]; studies in other populations are therefore warranted. Other Ascompounds such as arsenolipids and arsenosugars did not show enough potential as fish intake biomarkers because the biotransformation to larger organoarsenic metabolites does not always occur in the aquatic food chain. Moreover, in the human body, arsenosugars and arsenolipids are metabolized by the liver to thioarsenic [273] and As-containing fatty acids [274], respectively, rather than to DMAs or other smaller As-metabolites [267]. Inter-individual variability in arsenosugar metabolism has also been previously reported [273]. These As compounds are therefore unlikely to be good biomarker with specificity to fish or shellfish intake.

In conclusion, $\mathrm{n}-3$ LCPUFA in blood are valid BFI for the intake of fatty fish and fish oil at the individual level and would therefore reflect compliance to these foods in experimental studies, with measurements in different blood compartments reflecting the average intakes over different time periods. Marine lean fish and shellfish also contribute to their levels, while the contribution from freshwater fish depends on their feed. Total n-3 LCPUFA in most blood compartments should therefore reflect 
overall fish and shellfish intakes in populations with mixed diets. CMPF and related furan fatty acid metabolites in blood or urine are not sufficiently investigated; they may reflect more recent intakes of fish, fish oil, and shellfish but might also reflect habitual intakes over the last 4 weeks or more, and may depend on the origins of these foods. TMAO in urine is a shorter-term marker of intake for certain marine fish and shellfish, especially those from cold-temperate, deeper sea levels. Additional biomarkers of mainly marine fish and shellfish may include arsenobetaines in urine and possibly plasma, but further validation is needed for these markers, as well as for CMPF and TMAO. A biomarker reflecting intake of lean freshwater fish has not been found in this literature review. No specific biomarkers arose from our separate search on shellfish.

\section{Validation of candidate biomarkers}

The literature search outlined in the previous sections points to several putative biomarkers which may potentially be used to assess intake of animal protein, meat or fish and shellfish. However, several of these putative markers were not considered suitable as candidate BFIs for any of these foods. The reason for inclusion or exclusion of each compound from the list of putative markers is reported in Additional file 3: Table S4. The resulting list of candidate biomarkers have been further evaluated to assess their usefulness as BFIs through the eight validation criteria, previously proposed by the FoodBAll consortium [24] as reported in Table 1.

All the candidate markers in Table 1 are plausible (question 1) in the sense that their origin or presence in the food is well known or explained and also reasonably unique for the food group. Regarding the ability to quantitatively estimate the intake of a certain food, most of the candidate BFIs seem valid at the population level; even though a good dose-response relationship has been reported for several of the BFIs at the individual or group level (question 2), we still have little evidence for exact quantitation of individual food intake in samples from observational studies. Variation may be high due to the high variability in the concentration of precursors in different foods, to differences in absorption and metabolism, and to matrix effects caused by other dietary factors. The use of BFIs for quantitative assessment of food intakes should therefore be carefully considered for each specific study population, sample type, and for each specific intake assessment. For the remaining questions, the available evidence depends on the marker and its application as outlined below.

None of the meat exposures can be assessed quantitatively and without limitations by any candidate BFI. In general, the development of meat biomarkers faces significant challenges, such as (i) the overlap between meat-related metabolites and products of human muscle catabolism, (ii) variations in raw meat caused by variation between and possibly also within species, and (iii) the influence of production systems and feeding regimes. In spite of this, some BFIs are promising candidates and can be used under some circumstances for each of the exposures; these are outlined and discussed below, in light of the eight validation criteria. Table 1 should be used as an indicator of lacking evidence for each candidate BFI that needs to be addressed in further research.

Animal protein intake may be determined by the changes in isotope ratios, i.e., $\delta 15 \mathrm{~N}$ and/or $\delta 13 \mathrm{C}$ in urine, stool, hair or blood. Dose-response for both ratios has been observed for hair and whole blood. The repeated-dose time-response was established for hair samples for a single individual indicating that more than 5 months may be needed to change levels in hair close to the scalp. The time-course for blood has not been established, except that 1-4 weeks of dietary change seems insufficient. Time-response for urine and stool has not been established for single meals but for repeated intakes of diets based on animal or plant protein, and it was shown that 8 days of change was enough to alter the isotope ratios. The ratios in hair and blood are robust since they have been successfully applied in experimental as well as observational settings and they are reliable since they have repeatedly been validated against other intake measures, including dietary records and markers of aquatic meat intake. Robustness and reliability have not yet been established for the shorter-term markers in urine or stool. The ratios are chemically and biologically stable since stable isotopes cannot change with storage or handling and since they are known to work also in archeology. Moreover, their analytical performance is well established, their precision and accuracy is high, and inter-individual variability low, which has been observed in several laboratories, indicating also good reproducibility in terms of absolute values. The major weakness is the variation between populations due to food matrix effects and the lack of knowledge about the ability of the marker to pick up smaller differences in the average intakes of animal protein in individuals. Overall, the isotope ratios are excellent for comparing longer-term overall animal protein intakes within a population but need further refinement for measurements of shorter-term intakes or for estimating intakes accurately.

Total meat intake, including terrestrial and aquatic meats, may be assessed at the group level by 3-MH in blood or urine and potentially by anserine due to their presence in poultry and/or fish; the most promising markers of total meat intake at the individual level may be creatine in blood or urine or Hyp in blood. The latter (Hyp) may be a general marker of all meat since it reflects intake of cartilage, present also in offal. Doseresponse has been shown only for the markers in blood. The measurements in blood seem robust and have been 


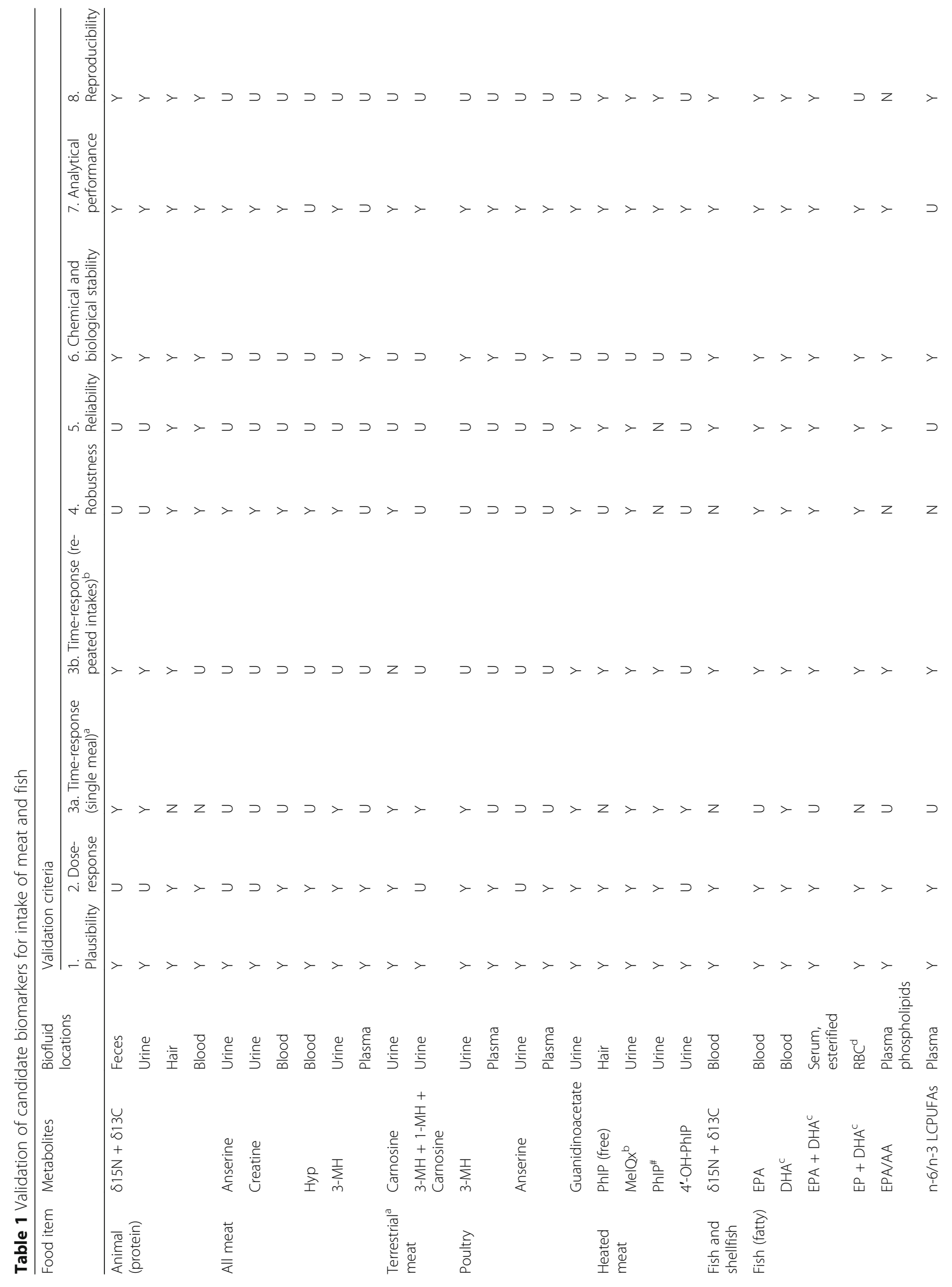




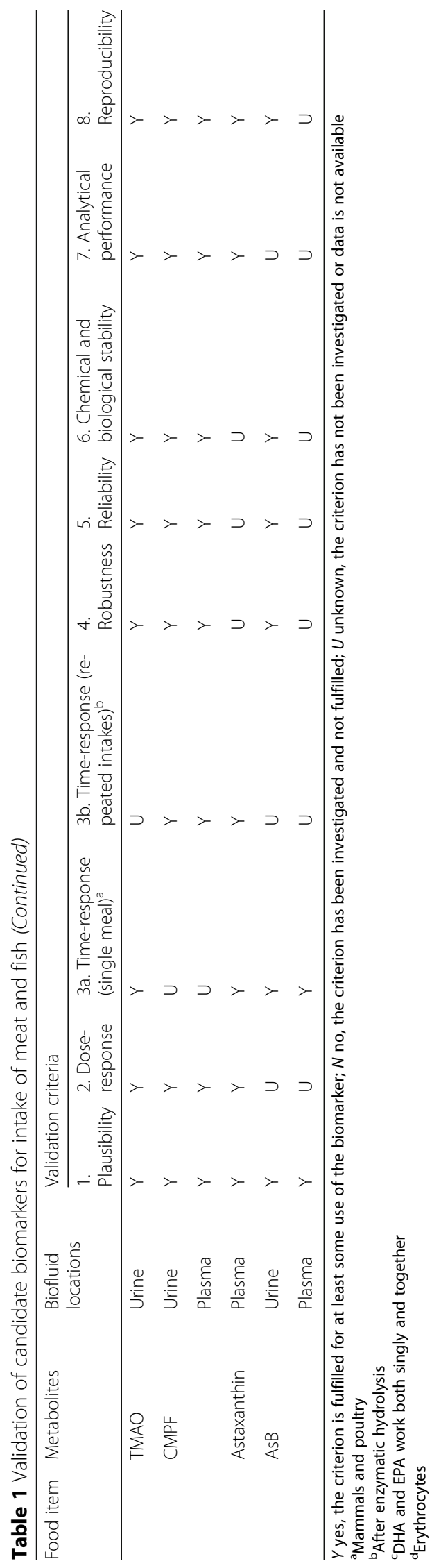


shown so far to associate with habitual intakes at the group level. Plasma Hyp has also been associated with meat intake in a few observational studies. Analytical performance seems adequate for creatine, while Hyp exists both free and as part of dipeptides and better validation is needed for each of these or for their combination. There are sparse data for validation of the individual variability, shorter- and longer-term time-response, reliability, and reproducibility of these markers. Urinary creatine has been proposed as a marker of habitual meat intake, with good robustness shown also in observational studies. Further validation studies are needed to evaluate reliability of these markers when different sources of meat are consumed.

Terrestrial meat (muscle) intake, including meat from mammals and birds, may be assessed by quantifying carnosine in urine. Carnosine seems to reflect only terrestrial meat intake, as it is absent in almost all aquatic meats. The marker has been reported to show dose-response and single meal time-response with no apparent accumulation upon repeated daily intakes; thus it is only reflecting recent meat intakes. The marker also seems robust in studies with a complex diet and it is strongly associated with other dietary assessment, i.e., FFQs at the group level, although there is still a paucity regarding the reliability at the individual level. Chemical and biological stability in urine and inter-laboratory reproducibility studies are also lacking while analytical performance seems adequate with LC-MS-based analytical platforms available for accurate measurements. Carnosine in combination with 1- and 3$\mathrm{MH}$, provided a better estimate of meat intake than each of the compounds alone [275]. Even though this model needs to be validated for reliability in observational studies, it underlines that measuring a combination of markers could optimize dietary assessment of meat intake.

Intake of white meat, i.e., poultry, but also some species of fish, may be assessed by the urinary excretion of 3-MH or anserine, or by 3-MH in plasma. Anserine is present in hen and turkey muscle and to a lesser extent in meat from several species of fish, including cod, salmon and tuna; excretion in humans may therefore mainly reflect poultry intake, while more studies are needed to assess the influence of fish on this marker. Human excretion of anserine has been shown to be strongly associated only with chicken intake and not with fish at the group level (high vs. nonconsumers) in a small European sub-study [38]. Anserine excretion after dietary intake has acceptable analytical performance in several analytical systems, stability after longer-term storage, and robustness; but experimental evidence for dose-response and time-response is still missing, including for experimental studies with fish intake. On the other hand, the validation of 3-MH in urine shows reproducible dose-, and time-response in experimental studies, although it is strongly associated with the intake of both bird and fish meat in observational studies. Increased excretion is also observed after red meat intake, albeit less strongly [276]; even so, experimental studies showing time- and dose-response of 3-MH excretion have been observed after intake of beef [43]. Inter-individual variation seems limited and background excretion rates with lactoovo-vegetarian diets are low. Stability is excellent and analytical performance is good in systems able to discriminate 1- and 3-MH [277, 278], but more data are needed regarding relative excretion levels after different sources of meat. Postprandial plasma 3-MH has been shown to respond stronger to chicken than to fish (haddock) with clear dose-response but with only marginal increases after high intakes of beef compared with other sources [38]. Plasma or serum 3-MH has not been extensively used as a marker in observational studies and most aspects of validation are therefore still missing, needing further research. From the scoring pattern, 3-MH in urine can be considered a valid compliance marker of poultry intake but may reflect general meat intake more broadly in samples from observational studies. Further validation studies are needed to study the relationship of 3-MH excretion with different kinds of aquatic meats.

Intake of cooked chicken may be quantified reliably by urinary levels of guanidinoacetate, which shows good dose-response, robustness, and analytical performance in a recent study from UK and Ireland [46] using NMR; guanidinoacetate in urine has also been observed in Denmark after a diet with mixed foods, including chicken [113]; however, further validation is needed in relation to other poultry meats and to potential geographical variation in the contents of this compound in chicken meat.

Average intake of heated muscle food may be assessed by the level of PhIP in hair while urinary excretion of PhIP, 4-OH-PhIP or MeIQx after hydrolysis of conjugates may be markers of recent intakes. All markers can be quantified reliably, while sample pretreatment (hydrolysis) strongly affects the measured concentrations. Dose-response was shown experimentally for PhIP in urine during a 4-day intervention showing little tendency for metabolite accumulation. However, urinary markers show large inter-individual variation. Only measurements of MeIQx in urine showed some agreement with FFQ-based estimations of cooked meat intakes in an observational study and MeIQx therefore seems as the more robust and reliable of the two. 4'-OH-PhIP may also be useful as a urinary marker of fried meat but lacks most aspects of validation, thus needing further research. Hair levels of PhIP show promise for estimating average intakes of fried and grilled meat over several months confirming levels in experimental diets with evidence of dose-response; however, robustness and reliability in observational studies have not yet been documented.

Habitual intake of aquatic meats, including fish and shellfish, or of fish oil, may be assessed by $n-3$ LCPUFA in 
whole blood, RBC, plasma or plasma phospholipids. Among the metabolites associated with repeated or habitual fish intake, EPA, DHA, and total n-3 LCPUFA have shown reasonable ability to discriminate between terrestrial meat and fish intake or to estimate consumption of fish, shellfish, and fish oil supplements. EPA and DHA levels in various fractions in blood have mainly been investigated after repetitive intakes, showing clear doseresponse with reported intakes; DHA was shown also to increase in plasma after a single meal in studies with cod or fish oil $[95,189]$, peaking at $2-4 \mathrm{~h}$. In experimental dietary studies, the time to reach steady state of LCPUFAs after fish, shellfish, or fish oil was 2-6 weeks for whole blood, plasma, and plasma lipids; 8 weeks for serum; and 4 weeks to 6 months for RBC. Total plasma free or esterified EPA and DHA were proved to be sufficiently robust and all analytical criteria are fulfilled.

Recent intake of aquatic meats, including fish and shellfish, may be assessed by several markers, including CMPF, AsB, or TMAO in urine, and CMPF or LCPUFA in plasma. CMPF and TMAO respond postprandially to meals with fish, showing dose-response and timeresponse. Complete excretion of TMAO within $24 \mathrm{~h}$ is observed in subjects with normal renal function. CMPF has a longer half-life in blood and is not completely excreted in $24 \mathrm{~h}$; in some groups of patients, the half-life may reach 4 weeks but better kinetic data are needed from experiments in healthy subjects. CMPF in blood seems to be observed in most cross-sectional studies of fish and shellfish intake while TMAO is not always associated with these intakes when cod or crustaceans are not an important part of the diets. CMPF seems therefore more reliable as a general fish marker in areas where furan fatty acids are present in the food chain but studies from additional areas of the world are needed to explore whether this is common everywhere. Both CMPF and TMAO are stable during storage, easily quantified but excretion is affected by kidney function, potentially making them reliable only in healthy subjects. Inter-laboratory comparison studies are not yet published but reported levels are comparable between laboratories, indicating fair reproducibility. $A s B$ has known short-term kinetics in plasma and urine but repeated-dose experimental studies are still lacking. The compound is likely to vary with geographical region and cooking practices but has been shown to be reliable in some regions, reflecting habitual intakes at the group level measured by dietary records. The analytical performance is sufficiently good to show clear kinetics by untargeted measurements but formal analytical validation is lacking for urine as well as for plasma. Plasma $A s B$ has been determined in a single experimental study. Much additional validation is therefore required for the use of AsB as a biomarker of aquatic meat intake.

\section{Conclusion}

A range of biomarkers have potential to estimate meat and fish intake. The $\delta 15 \mathrm{~N}$ and/or $\delta 13 \mathrm{C}$ ratios in hair are good determinants of long-term animal protein. Blood levels of creatine, hydroxyproline, and 3-MH are promising markers of total meat while carnosine works for terrestrial meats. However, more studies are needed to validate these markers, both alone and in combinations. The best validated markers are DHA and EPA in blood, serum, and blood lipids, clearly recognized as markers of habitual oily fish consumption. CMPF in blood and urine and TMAO in urine may also be useful markers to measure fish intake but depend on dietary habits. Overall, fish markers need validation in different populations. 3-MH seems to be a good biomarker of poultry intake showing dose-response, time-response, and good analytical performance but its robustness may be questioned because intake of some fish and possibly other meats are likely to interfere. Anserine is particularly good at characterizing poultry in both intervention and observational settings, but studies on time-response and dose-response are lacking. Guanidinoacetate excretion in urine is a promising new biomarker of chicken intake showing dose-response and robustness. No robust markers have been recognized so far to specifically assess red meat intake. BFIs able to discriminate between red and processed meats are also lacking. For fried and grilled meats, MeIQx in hydrolyzed urine and PhIP in hair are candidate biomarkers but need further work for their full validation. Additional studies on BFI discovery and validation are therefore needed before we can reliably assess or quantify intakes of all meat and most meat subgroups, except for aquatic n-3 LCPUFA.

\section{Supplementary information}

Supplementary information accompanies this paper at https://doi.org/10. 1186/s12263-019-0656-4.

Additional file 1: TableS1. Common keywords. TableS2. Specific keywords for each food group. Figure S1. Flow diagram of study selections describing each individual food group

Additional file 2: Table S3. Summary of the human studies reporting biomarkers positively related to intake of different kinds of meat, found in the systematic literature search. The studies are grouped and classified according to the category for which the biomarkers have been discussed in the main text.

Additional file 3: Table S4. Summary of the selected candidate biomarkers of meat and seafood intake and the discarded markers. For each marker, a brief explanation for inclusion or exclusion from the list of candidate FIBs is reported.

\footnotetext{
Abbreviations

1-OHP: 1-Hydroxypyrene; 1-OHPG: 1-Hydroxypyrene-O-glucuronide; 4'-OHPhIP: 2-Amino-1-methyl-6-(4'-hydroxyphenyl)imidazo[4,5-b]pyridine; 4-HNE: 4Hydroxy-2-nonenal; 5-OH-PhIP: 2-Amino-1-methyl-6-(5-

hydroxy)phenylimidazo[4,5-b]pyridine; 8-iso-PGF2A: 8-Iso-prostaglandin-

F2alpha; AA: Arachidonic acid; ALA: a-Linolenic acid; As: Arsenic;

AsB: Arsenobetaine; ATNC: Apparent total N-nitroso compounds;
} 
BFIs: Biomarker for food intake; CE: Cholesteryl esters; CMPF: 3-Carboxy-4methyl-5-propyl-2-furanpropionic acid; CVD: Cardiovascular disease: DHA: Docosahexaenoic acid; DHN-MA: 1,4-Dihydroxynonane mercapturic acid; DMA: Dimethylarsenate; DPA: Docosapentaenoic acid;

EPA: Eicosapentaenoic acid; EPIC: European Prospective Investigation into Cancer and Nutrition; FFQ: Food frequency questionnaires;

HAAs: Heterocyclic aromatic amines; Hyp: Hydroxyproline; LA: Linoleic acid; LCPUFAs: Long-chain polyunsaturated fatty acids;

LPE: Lysophosphatidylethanolamine; MelQx: 2-Amino-3,8dimethylimidazol4,5-f]quinoxaline; $\mathrm{MH}$ : Methylhistidine;

MMA: Monomethylarsenate; n-3: Omega-3; n-6: Omega-6; NAP: Naphthalene; NOC: N-nitroso compounds; PAHs: Polycyclic aromatic hydrocarbons; PC: Glycerophosphatidylcholine; PC: Phosphatidylcholine; PHE: Phenanthrene; PhIP: 2-Amino-1-methyl-6-phenylimidazo[4,5-b]pyridine; PhIP-M1: 7-Hydroxy5-methyl-3-phenyl-6,7,8,9-tetrahydropyrido[3',2':4,5]imidazo[1,2-a] pyrimidin-5ium chloride; Pro-Hyp: Prolyl-hydroxyproline; PUFA: Polyunsaturated fatty acids; RBC: Red blood cells; RCT: Randomized controlled trial; TBARS: Thiobarbituric acid reactive substances; THCC: 1,2,3,4-Tetrahydro- $\beta$ carboline-3-carboxylic acid; TMA: Trimethylamine; TMAO: Trimethylamine oxide

\section{Acknowledgments}

Not applicable.

\section{Authors' contributions}

CC and GP coordinated the work. GP performed the literature search for the general biomarkers of meat intake and heated meat and wrote the related sections; CC, SN, and KH performed the literature search for all the sections relative to fish and shellfish and wrote the related sections, except for astaxanthin that was written by MX; LH, CR, and LV carried out the literature search on biomarker for red, processed and offal meat, and wrote the related sections. SHP and LB performed the literature search on poultry and wrote that section. LOD revised and critically commented the manuscript and updated the searches and validation section until December 2018. CC and LOD thoroughly edited the manuscript. All the authors accepted the final version of the manuscript. All authors read and approved the final manuscript.

\section{Funding}

FoodBAll is a project funded by the BioNH call (grant number 529051002) under the Joint Programming Initiative, "A Healthy Diet for a Healthy Life." The project is funded nationally by the respective Research Councils; the work was funded in part by a grant from the Danish Innovation Foundation (\#4203-00002B) and a Semper Ardens grant from the Carlsberg Foundation to LOD; a postdoc grant from the University of Rome La Sapienza ("Borsa di studio per la frequenza di corsi o attività di perfezionamento all'estero" erogata ai sensi della legge 398/89) to GP; nationally funded by a grant from Science Foundation Ireland (SFI 14/JPI-HDHL/B3075) to SHP and LB; working grant from Juho Vainio Foundation, Saara Kuusisto and Salme Pennanen Grant for Cardiovascular Research and salary from Kuopio University Hospital (project number VTR 510RA17) to SN.

\section{Availability of data and materials}

Not applicable.

\section{Ethics approval and consent to participate}

Not applicable.

\section{Competing interests}

The authors declare no competing interests; however, LOD received funding in 2012 from the Danish meat industry to support work on meat intake biomarker development.

\section{Author details}

'Department of Nutrition, Exercise and Sports, University of Copenhagen, Rolighedsvej 30, 1958 Frederiksberg C, Denmark. ²Department of Veterinary Public Health \& Food Safety, Ghent University, Salisburylaan 133, 9820 Merelbeke, Belgium. ${ }^{3}$ School of Agriculture and Food Science, Institute of Food \& Health, University College Dublin, Belfield 4, Dublin, Ireland. ${ }^{4}$ Institute of Public Health and Clinical Nutrition, University of Eastern Finland, Yliopistonranta 1, 70210 Kuopio, Finland.
Received: 23 August 2019 Accepted: 12 November 2019 Published online: 30 December 2019

\section{References}

1. Wu G. Dietary protein intake and human health. Food Funct. 2016;7(3): 1251-65.

2. De Smet S, Vossen E. Meat: The balance between nutrition and health. A review. Meat Sci. 2016;120:145-56.

3. Lingbeck JM, Cordero P, O'Bryan CA, Johnson MG, Ricke SC, Crandall PG Functionality of liquid smoke as an all-natural antimicrobial in food preservation. Meat Sci. 2014;97(2):197-206.

4. Micha R, Wallace SK, Mozaffarian D. Red and processed meat consumption and risk of incident coronary heart disease, stroke, and diabetes mellitus: a systematic review and meta-analysis. Circulation. 2010;121(21):2271-83.

5. Abete I, Romaguera D, Vieira AR, Lopez de Munain A, Norat T. Association between total, processed, red and white meat consumption and all-cause, CVD and IHD mortality: a meta-analysis of cohort studies. Br J Nutr. 2014; 112(5):762-75.

6. Bouvard V, Loomis D, Guyton KZ, Grosse Y, Ghissassi FE, Benbrahim-Tallaa L, et al. Carcinogenicity of consumption of red and processed meat. Lancet Oncol. 2015;16(16):1599-600

7. Cross AJ, Ferrucci LM, Risch A, Graubard BI, Ward MH, Park Y, et al. A large prospective study of meat consumption and colorectal cancer risk: an investigation of potential mechanisms underlying this association. Cancer Res. 2010;70(6):2406-14.

8. WHO. Q\&A on the carcinogenicity of the consumption of red meat and processed meat. 2015. Available from: http://www.who.int/features/qa/ cancer-red-meat/en/.

9. Rohrmann S, Overvad K, Bueno-de-Mesquita HB, Jakobsen MU, Egeberg R, Tjønneland $\mathrm{A}$, et al. Meat consumption and mortality - results from the European Prospective Investigation into Cancer and Nutrition. BMC Med 2013;11(1):1-12.

10. Aune D, Ursin G, Veierød MB. Meat consumption and the risk of type 2 diabetes: a systematic review and meta-analysis of cohort studies. Diabetologia. 2009;52(11):2277-87.

11. Pan A, Sun Q, Bernstein AM, Schulze MB, Manson JE, Willett WC, et al. Red meat consumption and risk of type 2 diabetes: 3 cohorts of US adults and an updated meta-analysis. Am J Clin Nutr. 2011;94(4):1088-96.

12. Mari-Sanchis A, Gea A, Basterra-Gortari FJ, Martinez-Gonzalez MA, Beunza JJ, Bes-Rastrollo M. Meat consumption and risk of developing type 2 diabetes in the SUN project: a highly educated middle-class population. PLoS One. 2016;11(7):e0157990

13. Kris-Etherton PM, Harris WS, Appel L. Fish consumption, fish oil, omega-3 fatty acids, and cardiovascular disease. Circulation. 2002;106(21):2747-57.

14. World Cancer Research Fund / American Institute for Cancer Research. CUP Expert Report 2018: diet, nutrition, physicalactivity and colorectal cancer. 2018

15. Aglago EK, Huybrechts I, Murphy N, Casagrande C, Nicolas G, Pischon T, et al. Consumption of fish and long-chain n-3 polyunsaturated fatty acids is associated with reduced risk of colorectal cancer in a large European Cohort. Clin Gastroenterol Hepatol. 2019;\$1542-3565(19):30669-X.

16. Kaushik M, Mozaffarian D, Spiegelman D, Manson JE, Willett WC, Hu FB. Long-chain omega-3 fatty acids, fish intake, and the risk of type 2 diabetes mellitus. Am J Clin Nutr. 2009;90(3):613-20.

17. Patel PS, Sharp SJ, Luben RN, Khaw K-T, Bingham SA, Wareham NJ, et al. Association between type of dietary fish and seafood intake and the risk of incident type 2 diabetes. The European Prospective Investigation of Cancer (EPIC)-Norfolk cohort study. Diabetes Care. 2009;32(10):1857-63.

18. Du ZYZJ, Wang CR, Li LX, Man QQ, Lundebye AK, Froyland L. Risk-benefit evaluation of fish from Chinese markets: Nutrients and contaminants in 24 fish species from five big cities and related assessment for human health. Sci Total Environ. 2012;416:187-99.

19. Miklavčič A, Casetta A, Snoj Tratnik J, Mazej D, Krsnik M, Mariuz M, Sofianou K, Spirić Z, Barbone F, Horvat M. Mercury, arsenic and selenium exposure levels in relation to fish consumption in the Mediterranean area. Environ Res. 2013:120:7-17.

20. Dragsted LO. Biomarkers of meat intake and the application of nutrigenomics. Meat Sci. 2010;84(2):301-7.

21. Kipnis V, Subar AF, Midthune D, Freedman LS, Ballard-Barbash R, Troiano RP, et al. Structure of Dietary Measurement Error: Results of the OPEN Biomarker Study. Am J Epidemiol. 2003;158(1):14-21. 
22. Brunner E, Stallone D, Juneja M, Bingham S, Marmot M. Dietary assessment in Whitehall II: comparison of $7 \mathrm{~d}$ diet diary and food-frequency questionnaire and validity against biomarkers. Br J Nutr. 2001;86(3):405-14.

23. Praticò G, Gao Q, Scalbert A, Vergères G, Kolehmainen M, Manach C, et al. Guidelines for biomarker of food intake reviews (BFIRev): how to conduct an extensive literature search for biomarker of food intake discovery. Genes Nutr. 2018;13(1):3

24. Dragsted LO, Gao Q, Scalbert A, Vergères G, Kolehmainen M, Manach C, et al. Validation of biomarkers of food intake-critical assessment of candidate biomarkers. Genes Nutr. 2018;13(1):14

25. Münger LH, Garcia-Aloy M, Vázquez-Fresno R, Gille D, Rosana ARR, Passerini A, et al. Biomarker of food intake for assessing the consumption of dairy and egg products. Genes Nutr. 2018;13(1):26

26. Wishart DS, Tzur D, Knox C, Eisner R, Guo AC, Young N, et al. HMDB: the Human Metabolome Database. Nucleic Acids Res. 2007;35(suppl 1):D521-D6.

27. McNaught AD, Wilkinson A, IUPAC. Compendium of chemical terminology, 2nd ed. (the "Gold Book"). Oxford: Blackwell Scientific Publications; 1997.

28. Crush KG. Carnosine and related substances in animal tissues. Comp Biochem Physiol. 1970;34(1):3-30

29. Dunnett M, Harris RC. High-performance liquid chromatographic determination of imidazole dipeptides, histidine, 1-methylhistidine and 3methylhistidine in equine and camel muscle and individual muscle fibres. J Chromatogr B Biomed Sci Appl. 1997;688(1):47-55.

30. Harris $\mathrm{Cl}$, Milne $\mathrm{G}$. The occurrence of the $\mathrm{N}$ tau-methylhistidine-containing dipeptide, balenine, in muscle extracts of various mammals. Biochem Soc Trans. 1980;8(5):552.

31. Abe H, Emiko O, Hideo S, Akio M, Shohei Y. Human urinary excretion of Ihistidine-related compounds after ingestion of several meats and fish muscle. Int J Biochem. 1993;25(9):1245-9.

32. Abe H. Distribution of free I-histidine and related dipeptides in the muscle of fresh-water fishes. Comp Biochem Physiol B: Comp Biochem. 1983;76(1):35-9.

33. Gil-Agustí M, Esteve-Romero J, Carda-Broch S. Anserine and carnosine determination in meat samples by pure micellar liquid chromatography. J Chromatogr A. 2008;1189(1):444-50.

34. Block WD, Hubbard RW, Steele BF. Excretion of histidine and histidine derivatives by human subjects ingesting protein from different sources. J Nutr. 1965;85(4):419-25.

35. Mori M, Konoha-Mizuno K, Sadakane Y, Kawahara M. Carnosine concentration in the muscle of thoroughbred horses and its limitations in exercise performance. Trace Nutr Res. 2015;32:49-53.

36. Manhiani PS, Northcutt JK, Han I, Bridges WC, Dawson PL. Antioxidant activity of carnosine extracted from various poultry tissues. Poult Sci. 2013; 92(2):444-53.

37. Yeum K-J, Orioli M, Regazzoni L, Carini M, Rasmussen H, Russell RM, et al. Profiling histidine dipeptides in plasma and urine after ingesting beef, chicken or chicken broth in humans. Amino Acids. 2010;38(3):847-58.

38. Cheung W, Keski-Rahkonen P, Assi N, Ferrari P, Freisling H, Rinaldi S, et al. A metabolomic study of biomarkers of meat and fish intake. Am J Clin Nutr. 2017;105(3):600-8

39. Park YJ, Volpe SL, Decker EA. Quantitation of carnosine in humans plasma after dietary consumption of beef. J Agric Food Chem. 2005;53(12):4736-9.

40. Mitry P, Wawro N, Rohrmann S, Giesbertz P, Daniel H, Linseisen J. Plasma concentrations of anserine, carnosine and pi-methylhistidine as biomarkers of habitual meat consumption. Eur J Clin Nutr. 2018;73(5):692-702.

41. Datta SP, Harris H. Dietary origin of urinary methylhistidine. Nature. 1951; 168(4268):296-7.

42. Lloyd AJ, Beckmann M, Haldar S, Seal C, Brandt K, Draper J. Data-driven strategy for the discovery of potential urinary biomarkers of habitual dietary exposure. Am J Clin Nutr. 2013;97(2):377-89.

43. Cross AJ, Major JM, Sinha R. Urinary biomarkers of meat consumption. Cancer Epidemiol Biomark Prev. 2011;20(6):1107-11.

44. Sjolin J, Hjort G, Friman G, Hambraeus L. Urinary excretion of 1methylhistidine: a qualitative indicator of exogenous 3-methylhistidine and intake of meats from various sources. Metabolism. 1987;36(12):1175-84.

45. Wang Y, Gapstur SM, Carter BD, Hartman TJ, Stevens VL, Gaudet MM, et al. Untargeted metabolomics identifies novel potential biomarkers of habitual food intake in a cross-sectional study of postmenopausal women. J Nutr. 2018;148(6):932-43.

46. Yin X, Gibbons H, Rundle M, Frost G, McNulty BA, Nugent AP, et al. Estimation of chicken intake by adults using metabolomics-derived markers. J Nutr. 2017;147(10):1850-7 https://doi.org/10.3945/jn.117.252197.
47. Lloyd AJ, Favé G, Beckmann M, Lin W, Tailliart K, Xie L, et al. Use of mass spectrometry fingerprinting to identify urinary metabolites after consumption of specific foods. Am J Clin Nutr. 2011;94(4):981-91.

48. Myint T, Fraser GE, Lindsted KD, Knutsen SF, Hubbard RW, Bennett HW. Urinary 1-methylhistidine is a marker of meat consumption in Black and in White California Seventh-day Adventists. Am J Epidemiol. 2000;152(8):752-5.

49. Fraser GE, Jaceldo-Siegl K, Henning SM, Fan J, Knutsen SF, Haddad EH, et al. Biomarkers of dietary intake are correlated with corresponding measures from repeated dietary recalls and food-frequency questionnaires in the Adventist health study-2. J Nutr. 2016;146(3):586-94.

50. Kochlik B, Gerbracht C, Grune T, Weber D. The influence of dietary habits and meat consumption on plasma 3-methylhistidine-a potential marker for muscle protein turnover. Mol Nutr Food Res. 2018;62(9):e1701062. https://doi.org/10.1002/mnfr.201701062.

51. Ballard FJ, Tomas FM. 3-Methylhistidine as a measure of skeletal muscle protein breakdown in human subjects: the case for its continued use. Clin Sci. 1983;65(3):209.

52. Huszar G, Golenwsky G, Maiocco J, Davis E. Urinary 3-methylhistidine excretion in man: the role of protein-bound and soluble 3-methylhistidine. Br J Nutr. 1983:49(3):287-94.

53. Lukaski HC, Mendez J, Buskirk ER, Cohn SH. Relationship between endogenous 3-methylhistidine excretion and body composition. Am J Phys. 1981;240(3):E302-7.

54. Elia M, Carter A, Bacon S, Smith R. The effect of 3-methylhistidine in food on its urinary excretion in man. Clin Sci. 1980;59(6):509-11.

55. Khodorova NV, Rutledge DN, Oberli M, Mathiron D, Marcelo P, Benamouzig $R$, et al. Urinary metabolomics profiles associated to bovine meat ingestion in humans. Mol Nutr Food Res. 2018;63(1):e1700834.

56. Giesecke K, Magnusson I, Ahlberg M, Hagenfeldt L, Wahren J. Protein and amino acid metabolism during early starvation as reflected by excretion of urea and methylhistidines. Metabolism. 1989;38(12):1196-200.

57. Ross AB, Svelander C, Undeland I, Pinto R, Sandberg A-S. Herring and beef meals lead to differences in plasma 2-aminoadipic acid, $\beta$-alanine, 4hydroxyproline, cetoleic acid, and docosahexaenoic acid concentrations in overweight men. J Nutr. 2015;145(11):2456-63.

58. Schooneman MG, Vaz FM, Houten SM, Soeters MR. Acylcarnitines: reflecting or inflicting insulin resistance? Diabetes. 2013;62(1):1-8.

59. Stella C, Beckwith-Hall B, Cloarec O, Holmes E, Lindon JC, Powell J, et al. Susceptibility of human metabolic phenotypes to dietary modulation. J Proteome Res. 2006;5(10):2780-8.

60. Delanghe J, De Slypere JP, De Buyzere M, Robbrecht J, Wieme R, Vermeulen A. Normal reference values for creatine, creatinine, and carnitine are lower in vegetarians. ClinChem. 1989;35(8):1802.

61. O'Sullivan A, Gibney MJ, Brennan L. Dietary intake patterns are reflected in metabolomic profiles: potential role in dietary assessment studies. Am J Clin Nutr. 2011;93(2):314-21.

62. Playdon MC, Sampson JN, Cross AJ, Sinha R, Guertin KA, Moy KA, et al. Comparing metabolite profiles of habitual diet in serum and urine. Am J Clin Nutr. 2016;104(3):776-89.

63. Schmidt JA, Rinaldi S, Ferrari P, Carayol M, Achaintre D, Scalbert A, et al. Metabolic profiles of male meat eaters, fish eaters, vegetarians, and vegans from the EPIC-Oxford cohort. Am J Clin Nutr. 2015;102(6):1518-26.

64. Cederblad G. Effect of diet on plasma carnitine levels and urinary carnitine excretion in humans. Am J Clin Nutr. 1987;45(4):725-9.

65. Mitchell ME. Carnitine metabolism in human subjects. Am J Clin Nutr. 1978; 31(3):481-91.

66. Barr SI, Rideout CA. Nutritional considerations for vegetarian athletes. Nutrition. 2004;20(7):696-703.

67. Bertraml HC, Hoppe C, Petersen BO, Duus JØ, Mølgaard C, Michaelsen KF. An NMR-based metabonomic investigation on effects of milk and meat protein diets given to 8-year-old boys. Br J Nutr. 2007;97(4):758-63.

68. Lau CE, Siskos AP, Maitre L, Robinson O, Athersuch TJ, Want EJ, et al. Determinants of the urinary and serum metabolome in children from six European populations. BMC Med. 2018;16(1):202.

69. Pallister T, Jennings A, Mohney RP, Yarand D, Mangino M, Cassidy A, et al. Characterizing blood metabolomics profiles associated with self-reported food intakes in female twins. PLoS One. 2016;11(6):e0158568. https://doi. org/10.1371/journal.pone.0158568.

70. Pallister T, Sharafi M, Lachance G, Pirastu N, Mohney RP, MacGregor A, et al. Food Preference Patterns in a UK Twin Cohort. Twin Res Hum Genet. 2015; 18(6):793-805 
71. Rådjursöga M, Karlsson GB, Lindqvist HM, Pedersen A, Persson C, Pinto RC, et al. Metabolic profiles from two different breakfast meals characterized by 1H NMR-based metabolomics. Food Chem. 2017;231:267-74.

72. del Campo G, Gallego B, Berregi I, Casado JA. Creatinine, creatine and protein in cooked meat products. Food Chem. 1998;63(2):187-90.

73. Jacobsen JG, Smith LH. Biochemistry and physiology of taurine and taurine derivatives. Physiol Rev. 1968;48(2):424-511.

74. Xu J, Yang S, Cai S, Dong J, Li X, Chen Z. Identification of biochemical changes in lactovegetarian urine using 1H NMR spectroscopy and pattern recognition. Anal Bioanal Chem. 2010;396(4):1451-63.

75. Evered DF, Harvey MS, Luck L, Solari ME. The relationship between urinary taurine excretion and the intake of protein-rich foods. Life Sci. 1969;8(12): 601-5.

76. Khatibi N, Shahvazi S, Nadjarzadeh A, Samadi M, Zare F, Salehi-Abargouei A. Empirically derived dietary patterns and serum inflammatory markers in Iranian female teachers: A cross-sectional study. Nutr Diet. 2018;76(4): 462-71.

77. Ohara H, Matsumoto H, Ito K, Iwai K, Sato K. Comparison of quantity and structures of hydroxyproline-containing peptides in human blood after oral ingestion of gelatin hydrolysates from different sources. J Agric Food Chem. 2007:55(4):1532-5.

78. Acar E, Gurdeniz G, Khakimov B, Savorani F, Korndal SK, Larsen TM, et al. Biomarkers of individual foods, and separation of diets using untargeted LC MS-based plasma metabolomics in a randomized controlled trial. Mol Nut Food Res. 2019;63(1):e1800215. https://doi.org/10.1002/mnfr.201800215.

79. O'Brien DM. Stable isotope ratios as biomarkers of diet for health research Annu Rev Nutr. 2015;35:565-94.

80. O'Brien DM, Kristal AR, Jeannet MA, Wilkinson MJ, Bersamin A, Luick B. Red blood cell delta(15)N: a novel biomarker of dietary eicosapentaenoic acid and docosahexaenoic acid intake. Am J Clin Nutr. 2009:89(3):913-9.

81. Kuhnle GGC, Joosen A, Kneale CJ, O'Connell TC. Carbon and nitrogen isotopic ratios of urine and faeces as novel nutritional biomarkers of meat and fish intake. Eur J Nutr. 2013;52(1):389-95.

82. Patel PS, Cooper AJM, O'Connell TC, Kuhnle GGC, Kneale CK, Mulligan AM, et al. Serum carbon and nitrogen stable isotopes as potential biomarkers of dietary intake and their relation with incident type 2 diabetes: The EPICNorfolk study. Am J Clin Nutr. 2014;100(2):708-18.

83. Serra-Majem L, Nissensohn M, Overby NC, Fekete K. Dietary methods and biomarkers of omega 3 fatty acids: a systematic review. Br J Nutr. 2012;107: S64-76.

84. Yun HY, Lampe JW, Tinker LF, Neuhouser ML, Beresford SAA, Niles KR, et al. Serum nitrogen and carbon stable isotope ratios meet biomarker criteria for fish and animal protein intake in a controlled feeding study of a women's health initiative cohort. J Nutr. 2018;148(12):1931-7.

85. Nash SH, Bersamin A, Kristal AR, Hopkins SE, Church RS, Pasker RL, et al. Stable nitrogen and carbon isotope ratios indicate traditional and market food intake in an indigenous circumpolar population. J Nutr. 2012;142(1):84-90.

86. Petzke KJ, Boeing H, Klaus S, Metges CC. Carbon and nitrogen stable isotopic composition of hair protein and amino acids can be used as biomarkers for animal-derived dietary protein intake in humans. J Nutr. 2005;135(6):1515-20.

87. Petzke $\mathrm{K}$, Boeing $\mathrm{H}$, Metges CC. Choice of dietary protein of vegetarians and omnivores is reflected in their hair protein $13 \mathrm{C}$ and $15 \mathrm{~N}$ abundance. Rapid Commun Mass Spectrom. 2005;19(11):1392-400.

88. O'Connell TC, Hedges REM. Investigations into the effect of diet on modern human hair isotopic values. Am J Phys Anthropol. 1999;108(4):409-25.

89. Bol R, Pflieger C. Stable isotope (13C, $15 \mathrm{~N}$ and 34S) analysis of the hair of modern humans and their domestic animals. Rapid Commun Mass Spectrom. 2002;16(23):2195-200.

90. Nash SH, Kristal AR, Boyer BB, King IB, Metzgar JS, O'Brien DM. Relation between stable isotope ratios in human red blood cells and hair: implications for using the nitrogen isotope ratio of hair as a biomarker of eicosapentaenoic acid and docosahexaenoic acid. Am J Clin Nutr. 2009; 90(6):1642-7.

91. Koeth RA, Wang Z, Levison BS, Buffa JA, Org E, Sheehy BT, et al. Intestinal microbiota metabolism of $\mathrm{L}$-carnitine, a nutrient in red meat, promotes atherosclerosis. Nat Med. 2013;19(5):576-85.

92. Kruger R, Merz B, Rist MJ, Ferrario PG, Bub A, Kulling SE, et al. Associations of current diet with plasma and urine TMAO in the KarMeN study: direct and indirect contributions. Mol Nutr Food Res. 2017:61(11):e1700363. https://doi. org/10.1002/mnfr.201700363.
93. Andersen MBS, Reinbach HC, Rinnan A, Barri T, Mithril C, Dragsted LO. Discovery of exposure markers in urine for Brassica-containing meals served with different protein sources by UPLC-qTOF-MS untargeted metabolomics. Metabolomics. 2013:9(5):984-97.

94. Cho CE, Taesuwan S, Malysheva OV, Bender E, Tulchinsky NF, Yan J, et al. Trimethylamine-N-oxide (TMAO) response to animal source foods varies among healthy young men and is influenced by their gut microbiota composition: A randomized controlled trial. Mol Nutr Food Res. 2017;61(1): e1600324. https://doi.org/10.1002/mnfr.201600324.

95. Stanstrup J, Schou SS, Holmer-Jensen J, Hermansen K, Dragsted LO. Whey protein delays gastric emptying and suppresses plasma fatty acids and their metabolites compared to casein, gluten, and fish protein. J Proteome Res. 2014;13(5):2396-408

96. Wang Z, Bergeron N, Levison BS, Li XS, Chiu S, Jia X, et al. Impact of chronic dietary red meat, white meat, or non-meat protein on trimethylamine $\mathrm{N}$ oxide metabolism and renal excretion in healthy men and women. Eur Heart J. 2018:40(7):583-94.

97. Cade JE, Moreton JA, O'Hara B, Greenwood DC, Moor J, Burley VJ, et al. Diet and genetic factors associated with iron status in middle-aged women. Am J Clin Nutr. 2005;82(4):813-20.

98. Cox KA, Parkin PC, Anderson LN, Chen Y, Birken CS, Maguire JL, et al. Association between meat and meat-alternative consumption and iron stores in early childhood. Acad Pediatr. 2016;16(8):783-91.

99. Wittenbecher C, Muhlenbruch K, Kroger J, Jacobs S, Kuxhaus O, Floegel A, et al. Amino acids, lipid metabolites, and ferritin as potential mediators linking red meat consumption to type 2 diabetes. Am J Clin Nutr. 2015; 101(6):1241-50.

100. Felipe A, Guadalupe E, Perez D, et al. Serum ferritin is associated with metabolic syndrome and red meat consumption. Oxidative Med Cell Longev. 2015;2015:8.

101. Lewin MH, Bailey N, Bandaletova T, Bowman R, Cross AJ, Pollock J, et al. Red meat enhances the colonic formation of the DNA adduct O6Carboxymethyl guanine: implications for colorectal cancer risk. Cancer Res. 2006;66(3):1859-65.

102. Bastide NM, Chenni F, Audebert M, Santarelli RL, Tache S, Naud N, et al. A central role for heme iron in colon carcinogenesis associated with red meat intake. Cancer Res. 2015;75(5):870-9.

103. Joosen AM, Kuhnle GG, Aspinall SM, Barrow TM, Lecommandeur E, Azqueta A, et al. Effect of processed and red meat on endogenous nitrosation and DNA damage. Carcinogenesis. 2009;30(8):1402-7.

104. Bingham SA, Hughes R, Cross AJ. Effect of white versus red meat on endogenous $\mathrm{N}$-nitrosation in the human colon and further evidence of a dose response. J Nutr. 2002;132(11):3522S-5S.

105. Pierre F, Peiro G, Tache S, Cross AJ, Bingham SA, Gasc N, et al. New marker of colon cancer risk associated with heme intake: 1,4-dihydroxynonane mercapturic acid. Cancer Epidemiol Biomark Prev. 2006;15(11):2274-9.

106. Guillen MD, Goicoechea E. Toxic oxygenated alpha,beta-unsaturated aldehydes and their study in foods: a review. Crit Rev Food Sci Nutr. 2008; 48(2):119-36.

107. Massey R, Key PE. Examination of some fermented foods for the presence of apparent total N-nitroso compounds. Food Addit Contam. 1989;6(4):453-8.

108. Massey RC, Key PE, McWeeny DJ, Knowles ME. An investigation of apparent total N-nitroso compounds in beer. IARC Sci Publ. 1987;84:219-21.

109. Lee $L$, Archer MC, Bruce WR. Absence of volatile nitrosamines in human feces. Cancer Res. 1981;41(10):3992-4.

110. Holtrop G, Johnstone AM, Fyfe C, Gratz SW. Diet composition is associated with endogenous formation of $\mathrm{N}$-nitroso compounds in obese men. J Nutr. 2012;142(9):1652-8.

111. Sjölin J, Stjernström H, Henneberg S, Hambraeus L, Friman G. Evaluation of urinary 3-methylhistidine excretion in infection by measurements of 1 methylhistidine and the creatinine ratios. Am J Clin Nutr. 1989;49(1):62-70.

112. Michiels J, Maertens L, Buyse J, Lemme A, Rademacher M, Dierick NA, et al. Supplementation of guanidinoacetic acid to broiler diets: Effects on performance, carcass characteristics, meat quality, and energy metabolism. Poult Sci. 2012;91(2):402-12.

113. Schmedes M, Aadland EK, Sundekilde UK, Jacques H, Lavigne C, Graff IE, et al. Lean-seafood intake decreases urinary markers of mitochondrial lipid and energy metabolism in healthy subjects: Metabolomics results from a randomized crossover intervention study. Mol Nutr Food Res. 2016;60(7): $1661-72$. 
114. Majdeddin M, Golian A, Kermanshahi H, De Smet S, Michiels J. Guanidinoacetic acid supplementation in broiler chickens fed on cornsoybean diets affects performance in the finisher period and energy metabolites in breast muscle independent of diet nutrient density. Br Poult Sci. 2018;59(4):443-51.

115. Strickland PT, Qian Z, Friesen MD, Rothman N, Sinha R. Metabolites of 2amino-1-methyl-6-phenylimidazo(4,5-b)pyridine (PhIP) in human urine after consumption of charbroiled or fried beef. Mutat Res. 2002;506:163-73.

116. Stillwell WG, Sinha R, Tannenbaum SR. Excretion of the N(2)-glucuronide conjugate of 2-hydroxyamino-1-methyl-6-phenylimidazo[4,5-b]pyridine in urine and its relationship to CYP1A2 and NAT2 activity levels in humans. Carcinogenesis. 2002;23(5):831-8.

117. Liu G, Zong G, Hu FB, Willett WC, Eisenberg DM, Sun Q. Cooking methods for red meats and risk of type 2 diabetes: a prospective study of U.S. women. Diabetes Care. 2017:40(8):1041-9.

118. Andersen M-BS, Rinnan A, Manach C, Poulsen SK, Pujos-Guillot E, Larsen TM, et al. Untargeted metabolomics as a screening tool for estimating compliance to a dietary pattern. J Proteome Res. 2014;13(3):1405-18.

119. Sugimura T, Wakabayashi K, Nakagama H, Nagao M. Heterocyclic amines: mutagens/carcinogens produced during cooking of meat and fish. Cancer Sci. 2004;95(4):290-9.

120. Alaejos MS, Afonso AM. Factors that affect the content of heterocyclic aromatic amines in foods. Compr Rev Food Sci Food Saf. 2011;10(2):52-108.

121. Reistad R, Rossland OJ, Latva-Kala KJ, Rasmussen T, Vikse R, Becher G, et al. Heterocyclic aromatic amines in human urine following a fried meat meal. Food Chem Toxicol. 1997;35(10-11):945-55.

122. Turesky RJ, Le Marchand L. Metabolism and biomarkers of heterocyclic aromatic amines in molecular epidemiology studies: lessons learned from aromatic amines. Chem Res Toxicol. 2011;24(8):1169-214.

123. Teunissen SF, Rosing $H$, Schinkel AH, Schellens JHM, Beijnen JH. Review on the analysis of 2-amino-1-methyl-6-phenylimidazo[4,5-b]pyridine and its phase I and phase II metabolites in biological matrices, foodstuff and beverages. J Chromatogr B. 2010;878(31):3199-216.

124. Magagnotti C, Orsi F, Bagnati R, Celli N, Rotilio D, Fanelli R, et al. Effect of diet on serum albumin and hemoglobin adducts of 2-amino-1-methyl-6phenylimidazo[4,5-b]pyridine (PhIP) in humans. Int J Cancer. 2000;88(1):1-6.

125. Friesen MD, Rothman N, Strickland PT. Concentration of 2-amino-1-methyl6-phenylimidazo(4,5-b)pyridine (PhIP) in urine and alkali-hydrolyzed urine after consumption of charbroiled beef. Cancer Lett. 2001;173(1):43-51.

126. Stillwell WG, Kidd LCR, Wishnok JS, Tannenbaum SR, Sinha R. Urinary excretion of unmetabolized and phase II conjugates of 2-amino-1-methyl-6phenylimidazo 4,5-b pyridine and 2-amino-3,8-dimethylimidazo 4,5-f quinoxaline in humans: Relationship to cytochrome P4501A2 and Nacetyltransferase activity. Cancer Res. 1997;57(16):3457-64.

127. Turesky RJ, Gross GA, Stillwell WG, Skipper PL, Tannenbaum SR. Species differences in metabolism of heterocyclic aromatic amines, human exposure, and biomonitoring. Environ Health Perspect. 1994;102(SUPPL. 6): 47-51.

128. Kulp KS, Knize MG, Fowler ND, Salmon CP, Felton JS. PhIP metabolites in human urine after consumption of well-cooked chicken. J Chromatogr B Anal Technol Biomed Life Sci. 2004:802(1):143-53.

129. Knize MG, Kulp KS, Malfatti MA, Salmon CP, Felton JS. Liquid chromatography-tandem mass spectrometry method of urine analysis for determining human variation in carcinogen metabolism. J Chromatogr A. 2001;914(1-2):95-103.

130. Kulp KS, Knize MG, Malfatti MA, Salmon CP, Felton JS. Identification of urine metabolites of 2-amino-1-methyl-6-phenylimidazo 4,5-b pyridine following consumption of a single cooked chicken meal in humans. Carcinogenesis. 2000;21(11):2065-72.

131. Vanhaecke L, Knize MG, Noppe H, De Brabander H, Verstraete W, Van de Wiele T. Intestinal bacteria metabolize the dietary carcinogen 2-amino-1methyl-6-phenylimidazo 4,5-b pyridine following consumption of a single cooked chicken meal in humans. Food Chem Toxicol. 2008;46(1):140-8.

132. Busquets R, Frandsen H, Jonsson JA, Puignou L, Teresa Galceran M, Skog K. Biomonitoring of dietary heterocyclic amines and metabolites in urine by liquid phase microextraction: 2-amino-1-methyl-6-phenylimidazo 4,5-b pyridine (PhIP), a possible biomarker of exposure to dietary PhIP. Chem Res Toxicol. 2013:26(2):233-40.

133. Stillwell WG, Turesky RJ, Sinha R, Skipper PL, Tannenbaum SR. Biomonitoring of heterocyclic aromatic amine metabolites in human urine. Cancer Lett. 1999;143(2):145-8.
134. Ji H, Yu MC, Stillwell WG, Skipper PL, Ross RK, Henderson BE, et al. Urinary excretion of 2-amino-3,8-dimethylimidazo-[4,5-f]quinoxaline in white, black, and Asian men in Los Angeles County. Cancer Epidemiol Biomark Prev. 1994:3(5):407-11.

135. Kidd LC, Stillwell WG, Yu MC, Wishnok JS, Skipper PL, Ross RK, et al. Urinary excretion of 2-amino-1-methyl-6-phenylimidazo[4,5-b]pyridine (PhIP) in White, African-American, and Asian-American men in Los Angeles County. Cancer Epidemiol Biomark Prev. 1999;8(5):439-45.

136. Frandsen $\mathrm{H}$, Frederiksen $\mathrm{H}$, Alexander J. 2-amino-1-methyl-6-(5-hydroxy) phenylimidazo 4,5-b pyridine (5-OH-PhIP), a biomarker for the genotoxic dose of the heterocyclic amine, 2-amino-1-methyl-6-phenylimidazo 4, 5-b pyridine (PhIP). Food Chem Toxicol. 2002;40(8):1125-30.

137. Frandsen $\mathrm{H}$. Biomonitoring of urinary metabolites of 2-amino-1-methyl-6phenylimidazo 4,5-b pyridine (PhIP) following human consumption of cooked chicken. Food Chem Toxicol. 2008;46(9):3200-5.

138. Kataoka H, Inoue T, Ikekita N, Saito K. Development of exposure assessment method based on the analysis of urinary heterocyclic amines as biomarkers by on-line in-tube solid-phase microextraction coupled with liquid chromatography-tandem mass spectrometry Microextraction Techniques. Anal Bioanal Chem. 2014;406(8):2171-8.

139. Kataoka H, Inoue T, Saito K, Kato H, Masuda K. Analysis of heterocyclic amines in hair by on-line in-tube solid-phase microextraction coupled with liquid chromatography-tandem mass spectrometry. Anal Chim Acta. 2013; 786:54-60.

140. Fu Y, Zhao G, Wang S, Yu J, Xie F, Wang H, et al. Simultaneous determination of fifteen heterocyclic aromatic amines in the urine of smokers and nonsmokers using ultra-high performance liquid chromatography-tandem mass spectrometry. J Chromatogr A. 2014;1333:45-53.

141. Turesky RJ, Yuan J-M, Wang R, Peterson S, Yu MC. Tobacco smoking and urinary levels of 2-amino-9H-pyrido[2,3-b]indole in men of Shanghai, China. Cancer Epidemiol Biomarkers Prev. 2007;16(8):1554-60.

142. Pfau W, Skog K. Exposure to beta-carbolines norharman and harman. J Chromatogr B Anal Technol Biomed Life Sci. 2004;802(1):115-26.

143. Le Marchand L, Yonemori K, White KK, Franke AA, Wilkens LR, Turesky RJ. Dose validation of PhIP hair level as a biomarker of heterocyclic aromatic amines exposure: a feeding study. Carcinogenesis. 2016;37(7):685-91.

144. Kobayashi M, Hanaoka T, Hashimoto H, Tsugane S. 2-Amino-1-methyl-6phenylimidazo[4,5-b]pyridine (PhIP) level in human hair as biomarkers for dietary grilled/stir-fried meat and fish intake. Mutat Res. 2005;588(2):136-42.

145. Guo J, Yonemori K, Le ML, Turesky RJ. Method to biomonitor the cooked meat carcinogen 2-amino-1-methyl-6-phenylimidazo[4,5-b]pyridine in dyed hair by ultra-performance liquid chromatography-orbitrap high resolution multistage mass spectrometry. Anal Chem. 2015;87(12):5872-7.

146. Reistad R, Nyholm SH, Haug LS, Becher G, Alexander J. 2-amino-1-methyl-6phenylimidazo(4,5-beta)pyridine (PhIP), in human hair as biomarker for dietary exposure. Proc Am Assoc Cancer Res Ann Meet. 1999;40:151.

147. Bessette EE, Yasa I, Dunbar D, Wilkens LR, Marchand LL, Turesky RJ. Biomonitoring of carcinogenic heterocyclic aromatic amines in hair: a validation study. Chem Res Toxicol. 2009;22(8):1454-63.

148. Kobayashi M, Hanaoka T, Tsugane S. Validity of a self-administered food frequency questionnaire in the assessment of heterocyclic amine intake using 2-amino-l-methyl-6-phenylimidazo 4,5-b pyridine (PhIP) levels in hair. Mutat Res. 2007:630(1-2):14-9.

149. Turesky RJ, Liu L, Gu D, Yonemori KM, White KK, Wilkens LR, et al. Biomonitoring the cooked meat carcinogen 2-amino-1-methyl-6phenylimidazo 4,5-b pyridine in hair: impact of exposure, hair pigmentation, and cytochrome P450 1A2 phenotype. Cancer Epidemiol Biomark Prev. 2013;22(3):356-64

150. Turesky RJ, White KK, Wilkens LR, Le ML. Caffeine cytochrome P450 1A2 metabolic phenotype does not predict the metabolism of heterocyclic aromatic amines in humans. Chem ResToxicol. 2015;28(8):1603-15.

151. Boström C-E, Gerde P, Hanberg A, Jernström B, Johansson C, Kyrklund T, et al. Cancer risk assessment, indicators, and guidelines for polycyclic aromatic hydrocarbons in the ambient air. Environ Health Perspect. 2002;110(Suppl 3):451-88.

152. Zhang Y, Ding J, Shen G, Zhong J, Wang C, Wei S, et al. Dietary and inhalation exposure to polycyclic aromatic hydrocarbons and urinary excretion of monohydroxy metabolites - a controlled case study in Beijing, China. Environ Pollut. 2014;184:515-22.

153. Li Z, Mulholland JA, Romanoff LC, Pittman EN, Trinidad DA, Lewin MD, et al. Assessment of non-occupational exposure to polycyclic aromatic 
hydrocarbons through personal air sampling and urinary biomonitoring. J Environ Monit. 2010;12(5):1110-8.

154. Zhao L, Dong Y-H, Wang H. Residues of organochlorine pesticides and polycyclic aromatic hydrocarbons in farm-raised livestock feeds and manures in Jiangsu, China. Sci Total Environ. 2013;450-451:348-55.

155. Cross AJ, Sinha R. Meat-related mutagens/carcinogens in the etiology of colorectal cancer. Environ Mol Mutagen. 2004;44(1):44-55.

156. Li Z, Romanoff L, Bartell S, Pittman EN, Trinidad DA, McClean M, et al, Excretion profiles and half-lives of ten urinary polycyclic aromatic hydrocarbon metabolites after dietary exposure. Chem Res Toxicol. 2012; 25(7):1452-61

157. Jongeneelen FJ. Benchmark guideline for urinary 1-hydroxypyrene as biomarker of occupational exposure to polycyclic aromatic hydrocarbons. Ann Occup Hyg. 2001;45(1):3-13.

158. Kang DH, Rothman N, Poirier MC, Greenberg A, Hsu CH, Schwartz BS, et al. Interindividual differences in the concentration of 1-hydroxypyreneglucuronide in urine and polycyclic aromatic hydrocarbon-DNA adducts in peripheral white blood cells after charbroiled beef consumption. Carcinogenesis. 1995;16(5):1079-85.

159. Chien YC, Yeh CT. Amounts and proportion of administered pyrene dose excreted as urinary 1-hydroxypyrene after dietary exposure to polycyclic aromatic hydrocarbons. Arch Toxicol. 2010;84(10):767-76.

160. Viau C, Diakite A, Ruzgyte A, Tuchweber B, Blais C, Bouchard M, et al. Is 1 hydroxypyrene a reliable bioindicator of measured dietary polycyclic aromatic hydrocarbon under normal conditions? J Chromatogr B Anal Technol Biomed Life Sci. 2002;778(1-2):165-77.

161. Gunier RB, Reynolds P, Hurley SE, Yerabati S, Hertz A, Strickland P, et al. Estimating exposure to polycyclic aromatic hydrocarbons: A comparison of survey, biological monitoring, and geographic information system-based methods. Cancer Epidemiol Biomark Prev. 2006;15(7):1376-81.

162. Fagundes RB, Abnet CC, Strickland PT, Kamangar F, Roth MJ, Taylor PR, et al. Higher urine 1-hydroxy pyrene glucuronide (1-OHPG) is associated with tobacco smoke exposure and drinking mate in healthy subjects from Rio Grande do Sul, Brazil. BMC Cancer. 2006;6:139.

163. Islami F, Boffetta P, van Schooten FJ, Strickland P, Phillips DH, Pourshams A, et al. Exposure to polycyclic aromatic hydrocarbons among never smokers in Golestan province, Iran, an Area of High Incidence of Esophageal Cancer - a Cross-Sectional Study with Repeated Measurement of Urinary 1-OHPG in Two Seasons. Front Oncol. 2012;2:14.

164. Srogi K. Monitoring of environmental exposure to polycyclic aromatic hydrocarbons: a review. Environ Chem Lett. 2007;5(4):169-95.

165. Stich HF, Hornby AP, Dunn BP. The effect of dietary factors on nitrosoproline levels in human urine. Int J Cancer. 1984;33(5):625-8.

166. Chakradeo PP, Nair J, Bhide SV. Endogenous formation of N-nitrosoproline and other N-nitrosamino acids in tobacco users. Cancer Lett. 1994;86(2): 187-94.

167. Haorah J, Zhou L, Wang X, Xu G, Mirvish SS. Determination of total Nnitroso compounds and their precursors in frankfurters, fresh meat, dried salted fish, sauces, tobacco, and tobacco smoke particulates. J Agric Food Chem. 2001;49(12):6068-78.

168. Martins DA, Custodio L, Barreira L, Pereira H, Ben-Hamadou R, Varela J, et al. Alternative Sources of $n-3$ Long-Chain Polyunsaturated Fatty Acids in Marine Microalgae. Mar Drugs. 2013;11(7):2259-81.

169. Guiheneuf F, Stengel DB. LC-PUFA-enriched oil production by microalgae: accumulation of lipid and triacylglycerols containing n-3 LC-PUFA is triggered by nitrogen limitation and inorganic carbon availability in the marine haptophyte Pavlova lutheri. Mar Drugs. 2013;11(11):4246-66.

170. Anon. USDA Food Composition Databases. USDA National Nutrient Database for Standard Reference, Release 20. Apr 2018 ed. In: online United States Department of Agriculture, Agricultural Research Service; 2018.

171. Meyer BJ, Mann NJ, Lewis JL, Milligan GC, Sinclair AJ, Howe PR. Dietary intakes and food sources of omega- 6 and omega-3 polyunsaturated fatty acids. Lipids. 2003;38(4):391-8.

172. Nagasaka R, Gagnon C, Swist E, Rondeau I, Massarelli I, Cheung W, et al. EPA and DHA Status of South Asian and White Canadians Living in the National Capital Region of Canada. Lipids. 2014;49(10):1057-69.

173. Wall R, Ross RP, Fitzgerald GF, Stanton C. Fatty acids from fish: the antiinflammatory potential of long-chain omega-3 fatty acids. Nutr Rev. 2010; 68(5):280-9.

174. Williams CM, Burdge G. Long-chain $n-3$ PUFA: plant v. marine sources. Proc Nutr Soc. 2006;65(1):42-50.
175. Rajaram S, Haddad EH, Mejia A, Sabaté J. Walnuts and fatty fish influence different serum lipid fractions in normal to mildly hyperlipidemic individuals: A randomized controlled study. Am J Clin Nutr. 2009;89(5): 1657S-63S.

176. Saifullah A, Watkins BA, Saha C, Li Y, Moe SM, Friedman AN. Oral fish oil supplementation raises blood omega-3 levels and lowers C-reactive protein in haemodialysis patients- a pilot study. Nephrol Dial Transplant. 2007; 22(12):3561-7.

177. Marklund M, Magnusdottir OK, Rosqvist F, Cloetens L, Landberg R, Kolehmainen $\mathrm{M}$, et al. A dietary biomarker approach captures compliance and cardiometabolic effects of a healthy nordic diet in individuals with metabolic syndrome. J Nutr. 2014;144(10):1642-9.

178. Wu SY, Mayneris-Perxachs J, Lovegrove JA, Todd S, Yaqoob P. Fish-oil supplementation alters numbers of circulating endothelial progenitor cells and microparticles independently of eNOS genotype. Am J Clin Nutr. 2014; 100(5):1232-43.

179. Zabel R, Ash S, King N, Naslund E, Bauer J. Gender differences in the effect of fish oil on appetite, inflammation and nutritional status in haemodialysis patients. J Hum Nutr Diet. 2010;23(4):416-25.

180. Saadatian-Elahi M, Slimani N, Chajès V, Jenab M, Goudable J, Biessy C, et al. Plasma phospholipid fatty acid profiles and their association with food intakes: Results from a cross-sectional study within the European Prospective Investigation into Cancer and Nutrition. Am J Clin Nutr. 2009; 89(1):331-46.

181. Biltoft-Jensen A, Damsgaard CT, Andersen R, Ygil KH, Andersen EW, Ege M, et al. Accuracy of self-reported intake of signature foods in a school meal intervention study: Comparison between control and intervention period. Br J Nutr. 2015;114(4):635-44.

182. Okuda M, Sasaki S, Bando N, Hashimoto M, Kunitsugu I, Sugiyama S, et al. Carotenoid, tocopherol, and fatty acid biomarkers and dietary intake estimated by using a brief self-administered diet history questionnaire for older Japanese children and adolescents. J Nutr Sci Vitaminol. 2009;55(3): $231-41$.

183. Hautero U, Poussa T, Laitinen K. Simple dietary criteria to improve serum n3 fatty acid levels of mothers and their infants. Public Health Nutr. 2017; 20(3):534-41.

184. Dahl L, Maeland CA, Bjorkkjaer T. A short food frequency questionnaire to assess intake of seafood and n-3 supplements: validation with biomarkers. Nutr J. 2011;10:127.

185. Patterson AC, Hogg RC, Kishi DM, Stark KD. Biomarker and dietary validation of a Canadian food frequency questionnaire to measure eicosapentaenoic and docosahexaenoic acid intakes from whole food, functional food, and nutraceutical sources. J Acad Nutr Diet. 2012:112(7):1005-14.

186. Mina K, Fritschi L, Knuiman M. A valid semiquantitative food frequency questionnaire to measure fish consumption. Eur J Clin Nutr. 2007;61 (8): 1023-31.

187. Markhus MW, Graff IE, Dahl L, Seldal CF, Skotheim S, Braarud HC, et al. Establishment of a seafood index to assess the seafood consumption in pregnant women. Food Nutr Res. 2013;57. https://doi.org/10.3402/fnr.v57i0.1 9272.

188. Handeland K, Skotheim S, Baste V, Graff IE, Froyland L, Lie O, et al. The effects of fatty fish intake on adolescents' nutritional status and associations with attention performance: results from the FINS-TEENS randomized controlled trial. Nutr J. 2018;17(1):30.

189. Brown AJ, Pang E, Roberts DCK. Erythrocyte eicosapentaenoic acid versus docosahexaenoic acid as a marker for fish and fish oil consumptION. Prostaglandins Leukot Essent Fat Acids. 1991;44(2):103-6.

190. Patterson AC, Metherel AH, Hanning RM, Stark KD. The percentage of DHA in erythrocytes can detect non-adherence to advice to increase EPA and DHA intakes. Br J Nutr. 2014;111(2):270-8.

191. Hanwell HEC, Kay CD, Lampe JW, Holub BJ, Duncan AM. Acute Fish Oil and Soy Isoflavone Supplementation Increase Postprandial Serum (n-3) Polyunsaturated fatty acids and isoflavones but do not affect triacylglycerols or biomarkers of oxidative stress in overweight and obese hypertriglyceridemic Men. J Nutr. 2009;139(6):1128-34.

192. Browning LM, Walker CG, Mander AP, West AL, Madden J, Gambell JM, et al. Incorporation of eicosapentaenoic and docosahexaenoic acids into lipid pools when given as supplements providing doses equivalent to typical intakes of oily fish. Am J Clin Nutr. 2012;96(4):748-58.

193. Katan MB, Deslypere JP. vanBirgelen A, Penders M, Zegwaard M. Kinetics of the incorporation of dietary fatty acids into serum cholesteryl esters, 
erythrocyte membranes, and adipose tissue: an 18-month controlled study. J Lipid Res. 1997;38(10):2012-22.

194. Harris WS, Thomas RM. Biological variability of blood omega-3 biomarkers. Clin Biochem. 2010;43(3):338-40.

195. Arterburn LM, Hall EB, Oken H. Distribution, interconversion, and dose response of $\mathrm{n}-3$ fatty acids in humans. Am J Clin Nutr. 2006;83(6 Suppl): $1467 s-76 s$.

196. Cao J, Schwichtenberg KA, Hanson NQ, Tsai MY. Incorporation and clearance of omega-3 fatty acids in erythrocyte membranes and plasma phospholipids. Clin Chem. 2006;52(12):2265-72.

197. Patterson AC, Chalil A, Henao JJA, Streit IT, Stark KD. Omega-3 polyunsaturated fatty acid blood biomarkers increase linearly in men and women after tightly controlled intakes of $0.25,0.5$, and $1 \mathrm{~g} / \mathrm{d}$ of EPA plus DHA. Nutr Res. 2015;35(12):1040-51.

198. Metherel AH, Armstrong JM, Patterson AC, Stark KD. Assessment of blood measures of $n-3$ polyunsaturated fatty acids with acute fish oil supplementation and washout in men and women. Prostaglandins Leukot Essent Fat Acids. 2009;81(1):23-9.

199. Zheng JS, Lin M, Imamura F, Cai WW, Wang L, Feng JP, et al. Serum metabolomics profiles in response to $n-3$ fatty acids in Chinese patients with type 2 diabetes: a double-blind randomised controlled trial. Sci Rep. 2016;6:29522. https://doi.org/10.1038/srep29522.

200. Sala-Vila A, Cofán M, Mateo-Gallego R, Cenarro A, Civeira F, Ros E. Eicosapentaenoic acid in serum phospholipids relates to a less atherogenic lipoprotein profile in subjects with familial hypercholesterolemia. J Nutr Biochem. 2013;24(9):1604-8.

201. Gammelmark A, Madsen T, Varming K, Lundbye-Christensen S, Schmidt EB. Low-dose fish oil supplementation increases serum adiponectin without affecting inflammatory markers in overweight subjects. Nutr Res. 2012;32(1): 15-23.

202. Fukuoka Y, Nuruki N, Amiya S, Tofuku K, Aosaki S, Tsubouchi H. Effects of a fish-based diet and administration of pure eicosapentaenoic acid on brachial-ankle pulse wave velocity in patients with cardiovascular risk factors. J Cardiol. 2014;63(3):211-7.

203. Djuricic ID, Mazic SD, Kotur-Stevuljevic JM, Djordjevic VR, Sobajic SS. Longchain n-3 polyunsaturated fatty acid dietary recommendations are moderately efficient in optimizing their status in healthy middle-aged subjects with low fish consumption: A cross-over study. Nutr Res. 2014;34(3): 210-8.

204. Raatz SK, Rosenberger TA, Johnson LK, Wolters WW, Burr GS, Picklo MJ. Dose-dependent consumption of farmed atlantic salmon (salmo salar) increases plasma phospholipid n-3 fatty acids differentially. J Acad Nutr Diet. 2013;113(2):282-7.

205. Barceló-Coblijn G, Murphy EJ, Othman R, Moghadasian MH, Kashour T, Friel JK. Flaxseed oil and fish-oil capsule consumption alters human red blood cell $n-3$ fatty acid composition: A multiple-dosing trial comparing 2 sources of n-3 fatty acid. Am J Clin Nutr. 2008;88(3):801-9.

206. Flock MR, Skulas-Ray AC, Harris WS, Etherton TD, Fleming JA, Kris-Etherton PM. Determinants of erythrocyte omega-3 fatty acid content in response to fish oil supplementation: a dose-response randomized controlled trial. J Am Heart Assoc. 2013;2(6):e000513. https://doi.org/10.1161/JAHA.113.000513.

207. Flock MR, Skulas-Ray AC, Harris WS, Gaugler TL, Fleming JA, Kris-Etherton PM. Effects of supplemental long-chain omega-3 fatty acids and erythrocyte membrane fatty acid content on circulating inflammatory markers in a randomized controlled trial of healthy adults. Prostaglandins Leukot Essent Fat Acids. 2014;91(4):161-8.

208. Patterson AC, Chalil A, Aristizabal Henao JJ, Streit IT, Stark KD. Omega-3 polyunsaturated fatty acid blood biomarkers increase linearly in men and women after tightly controlled intakes of $0.25,0.5$, and $1 \mathrm{~g} / \mathrm{d}$ of EPA + DHA. Nutr Res. 2015;35(12):1040-51.

209. Von Schacky C, Fischer S, Weber PC. Long-term effects of dietary marine $\omega$ 3 fatty acids upon plasma and cellular lipids, platelet function, and eicosanoid formation in humans. J Clin Invest. 1985;76(4):1626-31.

210. Wallace FA, Miles EA, Calder PC. Comparison of the effects. of linseed oil and different doses of fish oil on mononuclear cell function in healthy human subjects. Br J Nutr. 2003;89(5):679-89.

211. Ulven SM, Kirkhus B, Lamglait A, Basu S, Elind E, Haider T, et al. Metabolic Effects of Krill Oil are Essentially Similar to Those of Fish Oil but at Lower Dose of EPA and DHA, in Healthy Volunteers. Lipids. 2011;46(1):37-46.

212. Hodson L, Crowe FL, McLachlan KJ, Skeaff CM. Effect of supplementation with flaxseed oil and different doses of fish oil for 2 weeks on plasma phosphatidylcholine fatty acids in young women. Eur J Clin Nutr. 2018;72(6): 832-40.

213. Zhang J, Wang C, Li L, Man Q, Song P, Meng L, et al. Inclusion of Atlantic salmon in the Chinese diet reduces cardiovascular disease risk markers in dyslipidemic adult men. Nutr Res. 2010;30(7):447-54.

214. Lindberg M, Midthjell K, Bjerve KS. Long-term tracking of plasma phospholipid fatty acid concentrations and their correlation with the dietary intake of marine foods in newly diagnosed diabetic patients: results from a follow-up of the HUNT Study, Norway. Br J Nutr. 2013;109(6):1123-34.

215. Bonaa KH, Bjerve KS, Nordoy A. Habitual fish consumption, plasma phospholipid fatty acids, and serum lipids: the Tromso study. Am J Clin Nutr. 1992;55(6):1126-34.

216. Amiano P, Dorronsoro M, de Renobales M, de Gordoa JCR, Irigoien I, Spain EG. Very-long-chain omega-3 fatty acids as markers for habitual fish intake in a population consuming mainly lean fish: the EPIC cohort of Gipuzkoa. Eur J Clin Nutr. 2001;55(10):827-32.

217. Crispim SP, Geelen A, Souverein OW, Hulshof PJM, Ruprich J, Dofkova M, et al. Biomarker-based evaluation of two 24-h recalls for comparing usual fish, fruit and vegetable intakes across European centers in the EFCOVAL Study. Eur J Clin Nutr. 2011;65(SUPPL. 1):S38-47.

218. Chung H, Nettleton JA, Lemaitre RN, Barr RG, Tsai MY, Tracy RP, et al. Frequency and Type of Seafood Consumed Influence Plasma (n-3) Fatty Acid Concentrations. J Nutr. 2008;138(12):2422-7.

219. Childs MT, Dorsett CS, King IB, Ostrander JG, Yamanaka WK. Effects of shellfish consumption on lipoproteins in normolipidemic men. Am J Clin Nutr. 1990;51(6):1020-7.

220. O'Dea K, Sinclair AJ. Increased proportion of arachidonic acid in plasma lipids after 2 weeks on a diet of tropical seafood. Am J Clin Nutr. 1982;36(5): 868-72.

221. Harris WS, Von Schacky C. The Omega-3 Index: A new risk factor for death from coronary heart disease? Prev Med. 2004;39(1):212-20.

222. Lindqvist HM, Langkilde AM, Undeland I, Sandberg AS. Herring (Clupea harengus) intake influences lipoproteins but not inflammatory and oxidation markers in overweight men. Br J Nutr. 2009;101(3):383-90.

223. Mann N, Sinclair A, Pille M, Johnson L, Warrick G, Reder E, et al. The effect of short-term diets rich in fish, red meat, or white meat on thromboxane and prostacyclin synthesis in humans. Lipids. 1997;32(6):635-44.

224. Lindqvist $H$, Langkilde AM, Undeland I, Radendal T, Sandberg AS. Herring (Clupea harengus) supplemented diet influences risk factors for CVD in overweight subjects. Eur J Clin Nutr. 2007;61(9):1106-13.

225. Din JN, Harding SA, Valerio CJ, Sarma J, Lyall K, Riemersma RA, et al. Dietary intervention with oil rich fish reduces platelet-monocyte aggregation in man. Atherosclerosis. 2008;197(1):290-6.

226. Zhang J, Wang C, Li L, Man Q, Meng L, Song P, et al. Dietary inclusion of salmon, herring and pompano as oily fish reduces CVD risk markers in dyslipidaemic middle-aged and elderly Chinese women. Br J Nutr. 2012; 108(8):1455-65.

227. Wu JH, Marchioli R, Silletta MG, Macchia A, Song X, Siscovick DS, et al. Plasma phospholipid omega-3 fatty acids and incidence of postoperative atrial fibrillation in the OPERA trial. J Am Heart Assoc. 2013;2(5):e000397. https://doi.org/10.1161/JAHA.113.000397.

228. Seierstad SL, Seljeflot I, Johansen O, Hansen R, Haugen M, Rosenlund G, et al. Dietary intake of differently fed salmon; the influence on markers of human atherosclerosis. Eur J Clin Investig. 2005;35(1):52-9.

229. Beblo S, Reinhardt H, Demmelmair H, Muntau AC, Koletzko B. Effect of fish oil supplementation on fatty acid status, coordination, and fine motor skills in children with phenylketonuria. J Pediatr. 2007;150(5):479-84.

230. Hals J, Bjerve KS, Nilsen H, Svalastog AG, Ek J. Essential fatty acids in the nutrition of severely neurologically disabled children. Br J Nutr. 2000;83(3):219-25.

231. Huang T, Li K, Asimi S, Chen Q, Li D. Effect of vitamin B-12 and n-3 polyunsaturated fatty acids on plasma homocysteine, ferritin, C-reactive protein, and other cardiovascular risk factors: a randomized controlled trial. Asia Pac J Clin Nutr. 2015;24(3):403-11.

232. Saldeen T, Wallin R, Marklinder I. Effects of a small dose of stable fish oil substituted for margarine in bread on plasma phospholipid fatty acids and serum triglycerides. Nutr Res. 1998;18(9):1483-92.

233. Soyland E, Funk J, Rajka G, Sandberg M, Thune P, Rustad L, et al. Effect of dietary supplementation with very-long-chain $\mathrm{n}-3$ fatty-acids in patients with psoriasis. N Engl J Med. 1993;328(25):1812-6.

234. Hanhineva $K$, Lankinen MA, Pedret A, Schwab U, Kolehmainen M, Paananen $J$, et al. Nontargeted metabolite profiling discriminates diet-specific 
biomarkers for consumption of whole grains, fatty fish, and bilberries in a randomized controlled trial. J Nutr. 2015;145(1):7-17.

235. Bondia-Pons I, Martinez JA, de la Iglesia R, Lopez-Legarrea P, Poutanen K, Hanhineva $\mathrm{K}$, et al. Effects of short- and long-term Mediterranean-based dietary treatment on plasma LC-QTOF/MS metabolic profiling of subjects with metabolic syndrome features: The Metabolic Syndrome Reduction in Navarra (RESMENA) randomized controlled trial. Mol Nutr Food Res. 2015; 59(4):711-28.

236. Playdon MC, Moore SC, Derkach A, Reedy J, Subar AF, Sampson JN, et al. Identifying biomarkers of dietary patterns by using metabolomics. Am J Clin Nutr. 2017;105(2):450-65.

237. Lu Y, Zou L, Su J, Tai ES, Whitton C, Dam RMV, et al. Meat and Seafood Consumption in Relation to Plasma Metabolic Profiles in a Chinese Population: A Combined Untargeted and Targeted Metabolomics Study. Nutrients. 2017;9(7). https://doi.org/10.3390/nu9070683.

238. Wahl HG, Tetschner B, Liebich HM. The effect of dietary fish oil supplementation on the concentration of 3-carboxy-4-methyl-5-propyl-2furanpropionic acid in human blood and urine. Hrc-J High Res Chrom. 1992; 15(12):815-8.

239. Tovar J, de Mello VD, Nilsson A, Johansson M, Paananen J, Lehtonen M, et al. Reduction in cardiometabolic risk factors by a multifunctional diet is mediated via several branches of metabolism as evidenced by nontargeted metabolite profiling approach. Mol Nutr Food Res. 2017;61(2):1600552.

240. Zhang AQ, Mitchell SC, Smith RL. Dietary precursors of trimethylamine in man: a pilot study. Food Chem Toxicol. 1999;37(5):515-20.

241. Wang Z, Klipfell E, Bennett BJ, Koeth R, Levison BS, DuGar B, et al. Gut flora metabolism of phosphatidylcholine promotes cardiovascular disease. Nature. 2011:472(7341):57-63.

242. Shiokawa Y, Misawa T, Date Y, Kikuchi J. Application of market basket analysis for the visualization of transaction data based on human lifestyle and spectroscopic measurements. Anal Chem. 2016;88(5):2714-9.

243. Yancey PH. Organic osmolytes as compatible, metabolic and counteracting cytoprotectants in high osmolarity and other stresses. J Exp Biol. 2005; 208(15):2819-30.

244. Tang WH, Wang Z, Levison BS, Koeth RA, Britt EB, Fu X, et al. Intestinal microbial metabolism of phosphatidylcholine and cardiovascular risk. $\mathrm{N}$ Engl J Med. 2013;368(17):1575-84.

245. Manor O, Zubair N, Conomos MP, Xu X, Rohwer JE, Krafft CE, et al. A multiomic association study of trimethylamine n-oxide. Cell Rep. 2018;24(4):935-46.

246. Herraiz T. Tetrahydro-beta-carbolines, potential neuroactive alkaloids, in chocolate and cocoa. J Agric Food Chem. 2000;48(10):4900-4.

247. Heinzmann SS, Merrifield CA, Rezzi S, Kochhar S, Lindon JC, Holmes E, et al. Stability and robustness of human metabolic phenotypes in response to sequential food challenges. J Proteome Res. 2012;11(2):643-55.

248. O'Gorman A, Morris C, Ryan M, O'Grada CM, Roche HM, Gibney ER, et al. Habitual dietary intake impacts on the lipidomic profile. J Chromatogr B Anal Technol Biomed Life Sci. 2014:966:140-6.

249. Odeberg JM, Lignell A, Pettersson A, Hoglund P. Oral bioavailability of the antioxidant astaxanthin in humans is enhanced by incorporation of lipid based formulations. Eur J Pharm Sci. 2003;19(4):299-304.

250. Hussein G, Sankawa U, Goto H, Matsumoto K, Watanabe H. Astaxanthin, a carotenoid with potential in human health and nutrition. J Nat Prod. 2006; 69(3):443-9.

251. Ambati RR, Phang SM, Ravi S, Aswathanarayana RG. Astaxanthin: Sources, extraction, stability, biological activities and its commercial applications-a review. Marine drugs. 2014;12(1):128-52.

252. Christiansen R, Lie O, Torrissen OJ. Growth and survival of Atlantic salmon, Salmo salar L., fed different dietary levels of astaxanthin. First-feeding fry. Aquac Nutr. 1995;1(3):189-98.

253. Coral-Hinostroza GN, Ytrestoyl T, Ruyter B, Bjerkeng B. Plasma appearance of unesterified astaxanthin geometrical $E / Z$ and optical $R / S$ isomers in men given single doses of a mixture of optical 3 and 3 'R/S isomers of astaxanthin fatty acyl diesters. Comp Biochem Physiol Toxicol Pharmacol. 2004;139(1-3):99-110.

254. Osterlie M, Bjerkeng B, Liaaen-Jensen S. Plasma appearance and distribution of astaxanthin $E / Z$ and $R / S$ isomers in plasma lipoproteins of men after single dose administration of astaxanthin. J Nutr Biochem. 2000;11(10):482-90.

255. Okada Y, Ishikura M, Maoka T. Bioavailability of astaxanthin in haematococcus algal extract: The Effects of Timing of Diet and Smoking Habits. Biosci Biotechnol Biochem. 2009;73(9):1928-32.
256. Miyazawa T, Nakagawa K, Kimura F, Satoh A, Miyazawa T. Plasma carotenoid concentrations before and after supplementation with astaxanthin in middle-aged and senior subjects. Biosci Biotechnol Biochem. 2011;75(9): 1856-8.

257. Karppi J, Rissanen TH, Nyyssonen K, Kaikkonen J, Olsson AG, Voutilainen S, et al. Effects of astaxanthin supplementation on lipid peroxidation. Int J Vitam Nutr. 2007;77(1):3-11.

258. Lorenz RT, Cysewski GR. Commercial potential for Haematococcus microalgae as a natural source of astaxanthin. Trends Biotechnol. 2000;18(4): $160-7$.

259. Park JS, Chyun JH, Kim YK, Line LL, Chew BP. Astaxanthin decreased oxidative stress and inflammation and enhanced immune response in humans. Nutr Metabol. 2010;7:18.

260. Res PT, Cermak NM, Stinkens R, Tollakson TJ, Haenen GR, Bast A, et al. Astaxanthin supplementation does not augment fat use or improve endurance performance. Med Sci Sports Exerc. 2013;45(6):1158-65.

261. Rufer CE, Moeseneder J, Briviba K, Rechkemmer G, Bub A. Bioavailability of astaxanthin stereoisomers from wild (Oncorhynchus spp.) and aquacultured (Salmo salar) salmon in healthy men: a randomised, double-blind study. Br J Nutr. 2008;99(5):1048-54.

262. Petyaev IM, Klochkov VA, Chalyk NE, Pristensky DV, Chernyshova MP, Kyle $\mathrm{NH}$, et al. Markers of Hypoxia and Oxidative Stress in Aging Volunteers Ingesting Lycosomal Formulation of Dark Chocolate Containing Astaxanthin. J Nutr Health Aging. 2018;22(9):1092-8.

263. Turunen AW, Mannisto S, Kiviranta H, Marniemi J, Jula A, Tiittanen $P$, et al. Dioxins, polychlorinated biphenyls, methyl mercury and omega-3 polyunsaturated fatty acids as biomarkers of fish consumption. Eur J Clin Nutr. 2010;64(3):313-23.

264. Domingo $J$, Bocio A. Levels of PCDD/PCDFs and PCBs in edible marine species and human intake: a literature review. Environ Int. 2007;33(3): 397-405.

265. Rahman MA, Hasegawa H, Lim RP. Bioaccumulation, biotransformation and trophic transfer of arsenic in the aquatic food chain. Environ Res. 2012;116: 118-35.

266. Lovreglio P, D'Errico MN, Gilberti ME, Drago I, Basso A, Apostoli P, et al. The influence of diet on intra and inter-individual variability of urinary excretion of arsenic species in Italian healthy individuals. Chemosphere. 2012;86(9): 898-905.

267. Molin M, Ulven SM, Dahl L, Telle-Hansen VH, Holck M, Skjegstad G, et al. Humans seem to produce arsenobetaine and dimethylarsinate after a bolus dose of seafood. Environ Res. 2012;112:28-39.

268. Lai WW, Sun Y, Ting E, Cullen WR, Reimer KJ. Arsenic speciation in human urine: are we all the same? Toxicol Appl Pharmacol. 2004;198(3):297-306.

269. Rivera-Nunez Z, Meliker JR, Meeker JD, Slotnick MJ, Nriagu JO. Urinary arsenic species, toenail arsenic, and arsenic intake estimates in a Michigan population with low levels of arsenic in drinking water. J Exp Sci Environ Epidemiol. 2012:22(2):182-90.

270. Navas-Acien A, Francesconi KA, Silbergeld EK, Guallar E. Seafood intake and urine concentrations of total arsenic, dimethylarsinate and arsenobetaine in the US population. Environ Res. 2011;111(1):110-8.

271. Panel EC. Scientific opinion on arsenic in food. EFSA J. 2009;7(10):1351.

272. Heitland P, Koster HD. Fast determination of arsenic species and total arsenic in urine by HPLC-ICP-MS: concentration ranges for unexposed german inhabitants and clinical case studies. J Anal Toxicol. 2008;32(4): 308-14

273. Raml R, Raber G, Rumpler A, Bauernhofer T, Goessler W, Francesconi KA. Individual variability in the human metabolism of an arsenic-containing carbohydrate, 2',3'-dihydroxypropyl 5-deoxy-5-dimethylarsinoyl-beta-Driboside, a naturally occurring arsenical in seafood. Chem Res Toxicol. 2009; 22(9):1534-40.

274. Schmeisser E, Goessler W, Francesconi KA. Human metabolism of arsenolipids present in cod liver. Anal Bioanal Chem. 2006:385(2):367-76.

275. Altorf-van der Kuil W, Brink EJ, Boetje M, Siebelink E, Bijlsma S, Engberink MF, et al. Identification of biomarkers for intake of protein from meat, dairy products and grains: a controlled dietary intervention study. Br J Nutr. 2013; 110(5):810-22.

276. O'Gorman A, Gibbons H, Brennan L. Metabolomics in the identification of biomarkers of dietary intake. Comput Struct Biotechnol J. 2013:4: e201301004

277. Houweling M, van der Drift SGA, Jorritsma R, Tielens AGM. Technical note: quantification of plasma 1- and 3-methylhistidine in dairy cows by high- 
performance liquid chromatography-tandem mass spectrometry. J Dairy Sci. 2012;95(6):3125-30.

278. Wang H, Hu P, Jiang J. Measurement of 1- and 3-methylhistidine in human urine by ultra performance liquid chromatography-tandem mass spectrometry. Clin Chim Acta. 2012;413(1):131-8.

\section{Publisher's Note}

Springer Nature remains neutral with regard to jurisdictional claims in published maps and institutional affiliations.

Ready to submit your research? Choose BMC and benefit from:

- fast, convenient online submission

- thorough peer review by experienced researchers in your field

- rapid publication on acceptance

- support for research data, including large and complex data types

- gold Open Access which fosters wider collaboration and increased citations

- maximum visibility for your research: over $100 \mathrm{M}$ website views per year

At BMC, research is always in progress. 